Communications in Physics, Vol. 24, No. 1 (2014), pp. 45-67

\title{
NEW ASPECTS ON STABILITY ANALYSIS OF A PLANAR CHARGE-VARYING COLLISIONAL DUST MOLECULAR CLOUD WITH FINITE THERMAL INERTIA
}

\author{
P. K. KARMAKAR AND B. BORAH \\ Department of Physics, Tezpur University, Napaam-784028, Tezpur, Assam, India \\ E-mail:pkk@tezu.ernet.in \\ Received 19 August 2013 \\ Accepted for publication 24 December 2013
}

\begin{abstract}
A theoretical evolutionary model for the nonlinear stability analysis of a planar dust molecular cloud (DMC) in quasi-neutral hydrodynamic equilibrium on the Jeans scales of space and time is developed. It is based on a selfgravitating multi-fluid model consisting of the warm electrons and ions, and the inertial cold dust grains with partial ionization. The Jeans assumption of self-gravitating uniform medium is adopted for fiducially analytical simplification by neglecting the zero-order field. So, the equilibrium is justifiably treated initially as "homogeneous", thereby validating nonlinear local analysis. The lowest-order finite inertial correction of the thermal species (thermal inertia, which is conventionally neglected), heavier grain-charge fluctuation and all the possible collisional dynamics are included simultaneously amid non-equilibrium plasma inhomogeneities. We apply a standard multiple scaling technique methodologically to show that the eigenmodes are collectively governed by a new electrostatic driven Korteweg-de Vries ( $d-K d V)$ equation having a self-consistent nonlinear driving source, and self-gravitational Korteweg-de Vries $(K d V)$ equation with neither a source, nor a sink. A detailed numerical shape-analysis with judicious multi-parameter variation parametrically portrays the excitation of electrostatic eigenmodes evolving as damped oscillatory shocks (nonconservative) with the increasing global amplitude due to the source, and extended two-tail compressive solitons (conservative), when the source-strength becomes very weak. In contrast, the self-gravitational counterparts grow as bell-shaped rarefactive soliton-like structures (conservative). The correlative effect of diverse plasma parameters on the amplitudes and patterns is explicitly investigated. Interestingly, this is conjectured that the grain-mass plays a key role in the eigenmode shaping (growth and decay) through the interplaying processes of pulsating gravito-electrostatic coupling. As the grain-mass increases, a new type of shock-to-soliton transition results, and so forth. The significance of the study in space, laboratory and astrophysical environments is stressed.
\end{abstract}

Keywords: nonlinear modes, KdV system, oscillatory shocks, soliton patterns.

\section{INTRODUCTION}

During the past few years, dusty plasmas have drawn considerable attention of researchers among plasma, astrophysics and space science communities [1-4]. This is because such plasmas support a great variety of waves, fluctuations and nonlinear coherent structures depending on the dust-grain nature having a great role to play in diverse areas of space, astrophysics including radiofrequency discharges, and so forth [4-8]. The existence of such eigenmode structures has many

(C)2014 Vietnam Academy of Science and Technology 
experimental supports as well [9-11]. Observations of such fluctuations and nonlinear coherent structures as solitons, shocks, double layers, etc. range widely from the normal laboratory scales [1-4,6-11] for a direct current (DC) glow discharge dusty plasma without self-gravity to the astrophysical scales [5,12-20] with self-gravitational effects under different plasma conditions having realistic significance in the formation mechanisms of stars, planets and other units of galactic structures.

The parametric signatures of the fluctuation patterns, eigenmodes and structures in selfgravitating dense dust molecular clouds (DMCs) on the astrophysical scale, so far is seen to the best of our knowledge, have been reported based on some purely idealistic model methodologies of zero-inertia of the plasma thermal species [12-20]. Recently, the nonlinear pulsational mode stability of such clouds with fluctuating grain-charge and the Boltzmann-distributed thermal species [21] has been analyzed. Later, a similar investigation with static grain-charge and finite thermal inertia in presence of weak frictional coupling of the neutral-charged grains [22] has also been proposed. However, it may be noted that none of the earlier authors addresses the effects on the eigenmode stability due to the consideration of the lowest-order finite inertia of the thermal species (hereafter, weak thermal inertia), grain-charge fluctuation, and all the possible collisional dynamics amid diverse inhomogeneities in such astrophysical dusty plasmas simultaneously. But, although weak, the weak thermal inertia [23-27] of the thermal species might have some roles to affect the co-excitation and co-evolution of the eigenmodes in wide spatially extended systems with all the above concurrent factors taken into account in different realistic situations. In addition, the grain usually acquires charge by capturing electrons and ions from the background plasma through collisional effects, while, looses charge by emitting photoelectrons induced by UV photons $[6,12-13]$. All these realistic influences, if considered collectively and simultaneously, might add some new effects to the eigenmode spectral structures and their related parametric dynamics, which is yet to be well investigated. Besides, the possible nature of new coupling processes of nonlinear convection due to fluidity, linear dispersion due to self-gravity, and dissipative mechanism due to various collisions in the system is still unknown.

In this paper, motivated by the curiosity of understanding the eigenmode structures under all the above realistic factors considered at a time, we try to develop a simple evolutionary model to study theoretically the nonlinear gravito-electrostatic eigenmodes in a planar one-dimensional (1-D) DMC in a quasi-neutral hydrodynamic equilibrium configuration on the Jeans scale. The key stimulus is to characterize the nature of the eigenmodes in presence of the thermal inertia of the cloud, grain-charge fluctuation, and various collisional effects incorporated at a time in a simplified manner amid non-equilibrium plasma inhomogeneities. The adopted technique is centered around a model based on the system of pair Korteweg-de Vries (KdV) equations. It is seen that the gravito-electrostatic eigenmode structures in the astrophysical charge-varying collisional grainy plasma appear mainly in the unique form of oscillatory shocks and solitary spectral patterns, which are subsequently shown to undergo some newer transitions in between the two distinct classes within some realistic parameter regime, particularly, dictated by the grain-mass. Lastly, this paper concentrates to discuss the main significance of such eigenmode structures lying in the diverse areas of cloud physics, space science and modern astrophysics because of their crucial role played in understanding self-gravitational collapse, formation and evolution of interstellar structure, star formation, galactic composition and its evolution, etc [12-22]. Besides, it may also be useful 
to help understanding of various other nonlinear waves, oscillations and fluctuation-spectra in different types of plasmas [28-32].

In addition to "introduction" part as described in Sec. I above, this paper is structurally organized as follows. Sec. II contains the physical model of the self-gravitating plasma system. Sec. III illustrates the basic governing equations of the cloud structure. Sec. IV portrays the derivation of nonlinear evolution equations, which is again divided into three subsections, IV.1, IV.2 and IV.3, depicting the electrostatic, self-gravitational and time-stationary fluctuations, respectively. Sec. V shows the numerical calculation scheme in two subsections, V.1 and V.2, showing the electrostatic fluctuations and self-gravitational counterparts, respectively. Sec. VI includes the relevant results and major discussions. Lastly and most importantly, Sec. VII presents the main conclusions of scientific interest and tentative astrophysical applicability along with some highlighted future directions focussingly.

\section{PHYSICAL MODEL}

A four-component simplified field-free planar DMC in astrophysical situation is considered under quasi-neutral hydrodynamic equilibrium configuration. This is well-known that the DMC equilibrium cannot be assumed like a truly homogeneous one, it must be derived from the basic governing structure equations themselves [13-14]. Still, for simplicity, hydrodynamic considerations are made to apply the conventional fluid equations for our investigation in presence of self-gravity contributed by the heavier grains.

The above model consideration is physically justifiable in accordance with the Jeans homogenization assumption of self-gravitating inhomogenous plasma for simplification [31-32]. Thus, the zero-order self-gravitational field is neglected and the equilibrium is treated initially as "homogeneous" which validates our nonlinear normal mode analysis (local). The plasma constituents are the thermal electrons, singly ionized positive ions, inertial spherical micron-sized neutral dust grains having partial ionization. The dust flow convections, collisional effects and grain-charge fluctuations are all taken into account in presence of the lowest-order inertial correction of the thermal species. This correction is systematically introduced through the modified Boltzmann population density distributions [26]. It may be pertinent to add further that high collisional momentum exchange from the plasma species to the gains may thermalize the complex plasma with the neutral grains. In addition, the self-gravitational effects are retained because of the massive grains existing in the cloud amid non-equilibrium plasma inhomogeneities.

The charging process of the grains is due basically to the attachment of the plasma species on the surface of the grains by the sticking mechanism [1-8]. The dynamics of the heavier neutral grains is merely governed by the Newtonian gravitational forces (unipolar) only, and that of the charged species is controlled by both the effective Columbic electrostatic (bipolar) and Newtonian gravitational forces. In the DMC, the two forces may become commensurable, i.e., $G m_{d}^{2} / q_{d}^{2} \sim 0(1)$. Thus, interplay between self-gravitational and electrostatic forces in the dynamics may lead to excitation of various nonlinear eigenmodes and novel phenomena in the astrophysical environment [5,12-20]. The grain-size is considered to be much smaller than the inter-grain separation, which is again lesser than the plasma Debye length. For our observation on the Jeans scales of space and time, the heavier grains are judiciously assumed to behave as an inertial fluid, whereas, the inertia-modified Boltzmann-populated electrons and ions are supposed to behave as thermal screening species, although slightly slowed down due to the inertial consideration. The 
efficacious cloud mass, as a whole, is collectively contributed by the heavier inertial grains (both neutral and charged), but within the validity limit of the Newtonian point-mass approximation [15-17]. For further simplicity, we ignore presence of rotation, kinematic viscosity, circulation, grain-size distribution, diverse anisotropies, magnetic field, etc.

\section{BASIC GOVERNING EQUATIONS}

The assumed hydrodynamic equilibrium of the proposed quasi-neutral cloud model is visualized as a quasi-static distribution of the multi-fluid constituent particles. The light neutral gas particles develop a constant background, which is weakly coupled to the collapsing charged grains. The equilibrium we consider for the fluctuation dynamics is governed by the collisional continuity, momentum, and the coupling electro-gravitational Poisson equations for the cloud constituents described with all the conventional notations [12] on the astrophysical scales of space and time. The dynamics of the plasma electrons in unnormalized form is described by

$$
\begin{gathered}
\frac{\partial n_{e}}{\partial t}+n_{e} \frac{\partial v_{e}}{\partial x}+v_{e} \frac{\partial n_{e}}{\partial x}=-f_{e d c}\left(n_{e}-n_{e o}\right), \\
T_{p} \frac{\partial n_{e}}{\partial x}+e n_{e} \frac{\partial \phi}{\partial x}+m_{e} n_{e} f_{e d c} v_{e}=0 .
\end{gathered}
$$

The dynamics of the plasma ions, similarly, is described by

$$
\begin{gathered}
\frac{\partial n_{i}}{\partial t}+n_{i} \frac{\partial v_{i}}{\partial x}+v_{i} \frac{\partial n_{i}}{\partial x}=-f_{i d c}\left(n_{i}-n_{i o}\right), \\
T_{p} \frac{\partial n_{i}}{\partial x}+e n_{i} \frac{\partial \phi}{\partial x}+m_{i} n_{i} f_{i d c} v_{i}=0 .
\end{gathered}
$$

The right hand sides of Eqs. (1) and (3) are the source or sink terms contributed by inelastic collisions of the respective thermal species with the dust particles [12]. Here, $f_{i d c}$ and $f_{e d c}$ are the ion-charged dust and electron-charged dust collision frequencies in unnormalized form, respectively. Since, we are interested in the low-frequency modes, we neglect the inertial terms in Eqs. (2) and (4) as usual. Also, the electrons and ions possess population densities $n_{e}, n_{i}$; velocities $v_{e}, v_{i}$; masses $m_{e}, m_{i}$; charges $-e,+e$; and temperatures $T_{e}, T_{i}$ such that $T_{e} \approx T_{i}=T_{p}$ (in eV); respectively. The equations describing the neutral grain dynamics are given as follows

$$
\begin{gathered}
\frac{\partial n_{d n}}{\partial t}+n_{d n} \frac{\partial v_{d n}}{\partial x}+v_{d n} \frac{\partial n_{d n}}{\partial x}=0 \\
m_{d n} n_{d n}\left[\frac{\partial v_{d n}}{\partial t}+v_{d n} \frac{\partial v_{d n}}{\partial x}\right]=-T_{p} \frac{\partial n_{d n}}{\partial x}-m_{d n} n_{d n} \frac{\partial \psi}{\partial x}-m_{d n} n_{d n} f_{n c}\left(v_{d n}-v_{d c}\right) .
\end{gathered}
$$

Furthermore, the dynamics of the charged grains with all usual notations is given by

$$
\begin{gathered}
\frac{\partial n_{d c}}{\partial t}+n_{d c} \frac{\partial v_{d c}}{\partial x}+v_{d c} \frac{\partial n_{d c}}{\partial x}=0 \\
m_{d c} n_{d c}\left[\frac{\partial v_{d c}}{\partial t}+v_{d c} \frac{\partial v_{d c}}{\partial x}\right]=-T_{p} \frac{\partial n_{d c}}{\partial x}-q_{d} n_{d c} \frac{\partial \phi}{\partial x}-m_{d c} n_{d c} \frac{\partial \psi}{\partial x}-m_{d c} n_{d c} f_{c n}\left(v_{d c}-v_{d n}\right),
\end{gathered}
$$

where, $f_{c n}$ and $f_{n c}$ are charged-neutral dust and neutral-charged dust collision frequencies, respectively. The spatial distributions of the electrostatic potential $(\phi)$ and self-gravitational potential $(\psi)$ 
in presence of the finite thermal inertia are defined by the combining Poisson equations as given below,

$$
\begin{gathered}
\frac{\partial^{2} \phi}{\partial x^{2}}=-4 \pi\left[e\left(n_{i}-n_{e}\right)-q_{d} n_{d c}\right] \\
\frac{\partial^{2} \psi}{\partial x^{2}}=4 \pi G\left(m_{d n} n_{d n}+m_{d c} n_{d c}-m_{d} n_{d o}+m_{e} n_{e}+m_{i} n_{i}\right) .
\end{gathered}
$$

By invoking the Jeans swindle [14-18] through $m_{d} n_{d 0}$ in Eq. (10), we would neglect the zeroth-order self-gravitational field, so that the equilibrium may, justifiably, be considered initially as homogeneous. Even so, the equilibrium force of gravity could be unimportant to affect the equilibrium dynamics within the domain of uniform cloud mass distribution. Indeed, a spatially homogeneous self-gravitating plasma system cannot be in static equilibrium (for which $\partial^{2} \psi / \partial \xi^{2} \sim 0$ ), since there is no pressure gradient to balance the gravitational force (originating from the equilibrium cloud-material distribution $m_{d n} n_{d n}+m_{d c} n_{d c}+m_{e} n_{e}+m_{i} n_{i} \approx m_{d} n_{d o}$ as evident from Eq. (10)). This physically means that self-gravitational potential is sourced only by density fluctuations of the infinite uniform homogeneous background medium under consideration [31-32]. The Jeans assumption (ad hoc) for the self-gravitating uniform homogeneous medium may not be the most suitable one, but it allows us to treat the self-gravitating inhomogeneous plasma dynamics analytically in a simplified way by neglecting the zero-order field so that local analyses could be carried out [31]. The results on the fluctuations and eigenmodes based on this homogenization assumption in most of the cases have been found to be not far from realistic picture [5, 7, 14-17, 31-32]. Now, if this self-gravitational symmetry of uniformity is broken either due to some mass fluctuations (purely neutral dust mass), or even due to the charge fluctuations (charged mass), the gravitational collapse will set in and evolve in a different manner resulting in the corresponding eigenmode spectra than those as conventionally discussed in the literature. Lastly, the charge dynamic equation [12] for the charge-fluctuated dust grains is given by

$$
\frac{\partial q_{d}}{\partial t}+v_{d c} \frac{\partial q_{d}}{\partial x}=e\left[f_{e d} \frac{\left(n_{e}-n_{e o}\right)}{n_{d o}}-f_{i d} \frac{\left(n_{i}-n_{e o}\right)}{n_{d o}}\right] .
$$

Here, $f_{e d}$ and $f_{i d}$ are electron-dust and ion-dust collision frequencies, respectively. The equilibrium dust density is $n_{d o}=n_{d c o}+n_{d n o}$. Here, $G\left(=6.67 \times 10^{-11} \mathrm{~N} \mathrm{~m}^{2} \mathrm{~kg}^{-2}\right)$ represents the universal gravitational constant. Furthermore, the neutral and charged grains each with mass $m_{d}$ and temperature $T_{d}$ have population densities $n_{d n}, n_{d c}$; and velocities $v_{d n}, v_{d c}$; respectively.

\section{NORMALIZED SET OF BASIC EQUATIONS}

We are interested in the application of the lowest-order inertial correction [23-27] of the plasma thermal species incorporated through the inertia-modified Boltzmann distributions [26] formulated on the Jeans scale. For simplicity, a standard normalization procedure [17] of the relevant astrophysical parameters is adopted. But, as is well-known, the self-gravitating plasmas are known to be inhomogeneous in nature, and thus, adopting constant normalization parameter values of the cloud variables throughout, which may vary from point to point spatiotemporally due to large-scale dynamics, is not so justifiable [14]. Therefore, the Jeans assumption of homogeneous medium [31-32], paving the way for a local-type nonlinear normal mode analysis [5,16-18,3132 ] in the process of mathematical simplification is adopted in our model. Accordingly, the new 
Boltzmann population density distributions specifically developed considering the weak inertia of the thermal electrons and ions in normalized form are given as,

$$
N_{e}=\exp \left[\left(\frac{1}{2}\right) \frac{m_{e}}{m_{i}} M_{e o}^{2}\{1-\exp (-2 \Phi)\}+\Phi\right],
$$

and

$$
N_{i}=\exp \left[\left(\frac{1}{2}\right) \frac{m_{i}}{m_{d}} M_{i o}^{2}\{1-\exp (2 \Phi)\}-\Phi\right] .
$$

It is clearly seen that the presence of the mass-ratios in the above expressions describes the active inertial roles of the thermal species in their relevant response time scales, which would affect the equilibrium and fluctuation dynamics of the grainy plasma model. If one considers $m_{e} / m_{i}, m_{i} / m_{d} \rightarrow 0$ for the inertialess electrons and ions in Eqs. (12)-(13), they reduce back to the normal inertialess Boltzmann distributions [12-13,15-16]. We are interested to see the effect of the population distributions given by Eqs. (12)-(13) on the electron and ion dynamics contributing to the stability behavior under investigation. Thus, it may be seen after substitution that for the electron description, there are two unknown variables $\left(M_{e}, \Phi\right)$ governed by two equations. Similarly, for the ion description as well, there are two unknown variables $\left(M_{i}, \Phi\right)$ described by two equations as the following. This is how the situation of over-determined set of unknowns for the electron and ion descriptions is avoided. Now, Eqs. (1)-(11) in normalized form are respectively enlisted as below,

$$
\begin{aligned}
& \frac{\partial N_{e}}{\partial \tau}+N_{e} \frac{\partial M_{e}}{\partial \xi}+M_{e} \frac{\partial N_{e}}{\partial \xi}=-F_{e d c}\left(N_{e}-1\right), \\
& \frac{\partial N_{e}}{\partial \xi}+N_{e} \frac{\partial \Phi}{\partial \xi}+\left(\frac{m_{e}}{m_{d}}\right) N_{e} M_{e} F_{e d c}=0, \\
& \frac{\partial N_{i}}{\partial \tau}+N_{i} \frac{\partial M_{i}}{\partial \xi}+M_{i} \frac{\partial N_{i}}{\partial \xi}=-F_{i d c}\left(N_{i}-1\right), \\
& \frac{\partial N_{i}}{\partial \xi}+N_{i} \frac{\partial \Phi}{\partial \xi}+\left(\frac{m_{i}}{m_{d}}\right) N_{i} M_{i} F_{i d c}=0 \\
& \frac{\partial N_{d n}}{\partial \tau}+N_{d n} \frac{\partial M_{d n}}{\partial \xi}+M_{d n} \frac{\partial N_{d n}}{\partial \xi}=0, \\
& N_{d n}\left[\frac{\partial M_{d n}}{\partial \tau}+M_{d n} \frac{\partial M_{d n}}{\partial \xi}\right]=-\frac{1}{n_{d n o}} \frac{\partial N_{d n}}{\partial \xi}-\left(\frac{m_{d n}}{n_{d n o} e}\right) N_{d n} \frac{\partial \Psi}{\partial \xi}-\left(\frac{1}{n_{d n o}}\right) F_{n c}\left(M_{d n}-M_{d c}\right), \\
& \frac{\partial N_{d c}}{\partial \tau}+N_{d c} \frac{\partial M_{d c}}{\partial \xi}+M_{d c} \frac{\partial N_{d c}}{\partial \xi}=0, \\
& N_{d c}\left[\frac{\partial M_{d c}}{\partial \tau}+M_{d c} \frac{\partial M_{d c}}{\partial \xi}\right]=-\frac{1}{n_{d c o}} \frac{\partial N_{d c}}{\partial \xi}-\left(\frac{q_{d}}{n_{d c o}}\right) Q_{d} N_{d c} \frac{\partial \Phi}{\partial \xi} \\
& -\left(\frac{m_{d c}}{n_{d c o} e}\right) N_{d c} \frac{\partial \Psi}{\partial \xi}-\left(\frac{1}{n_{d c o}}\right) F_{c n}\left(M_{d c}-M_{d n}\right) \text {, } \\
& \frac{\partial^{2} \Phi}{\partial \xi^{2}}=\frac{e}{m_{d c}^{2} n_{d o} G}\left[e\left(n_{e o} N_{e}-n_{i o} N_{i}\right)+\left(q_{d o} n_{d c o}\right) Q_{d} N_{d c}\right], \\
& \frac{\partial^{2} \Psi}{\partial \xi^{2}}=\frac{e}{m_{d n} n_{d o}}\left\{n_{d n o} N_{d n}+n_{d c o} N_{d c}-n_{d o} N_{d o}+n_{e o}\left(\frac{m_{e}}{m_{d}}\right) N_{e}+n_{i o}\left(\frac{m_{i}}{m_{d}}\right) N_{i}\right\},
\end{aligned}
$$




$$
\frac{\partial Q_{d}}{\partial \tau}+M_{d c} \frac{\partial Q_{d}}{\partial \xi}=\frac{e}{q_{d o}}\left(\frac{n_{e o}}{n_{d o}}\right) F_{e d}\left[\left\{N_{e}-\left(\frac{n_{i o}}{n_{e o}}\right)\left(\frac{F_{i d}}{F_{e d}}\right) N_{i}\right\}-\left(1+\frac{n_{i o}}{n_{e o}} \frac{F_{i d}}{F_{e d}}\right)\right] .
$$

As already mentioned, the Jeans swindle [13-16, 31] is invoked in the derivation of Eq. (23), which is in fact a kind of local approximation for equilibrium mass distribution as in the case of quasi-neutral equilibrium charge distribution. Moreover, the independent coordinates like position $(\xi)$ and time $(\tau)$ are normalized by the Jeans length $\left(\lambda_{J}\right)$ and Jeans time $\left(\omega_{J}^{-1}\right)$ scales, respectively. The parameters $M_{e}(\xi), M_{i}(\xi), M_{d n}(\xi)$, and $M_{d c}(\xi)$ represent the flow velocities of the electrons, ions, neutral and charged grains normalized by the dust sound phase speed $C_{s s}$ each. Moreover, $N_{e}, N_{i}, N_{d n}$ and $N_{d c}$ are the population densities of the electrons, ions, neutral and charged grains normalized by their equilibrium densities $n_{e 0}, n_{i 0}, n_{d n 0}$, and $n_{d c 0}$, respectively. Both the electrostatic potential $\Phi$ and self-gravitational potential $\Psi$ are normalized by the same electron thermal potential $T_{p} / e$ so as to compare their fluctuation levels on a common equivalent unit. The grain charge $Q_{d}$ is normalized by the equilibrium grain charge $q_{d 0}$. Lastly, $F_{e d c}, F_{e d}$, $F_{i d c}, F_{i d}, F_{n c}$ and $F_{c n}$ are the collision frequencies of the electrons and charged grains; electrons and dust grains; ions and charged grains; ions and dust grains; neutral and charged grains; and finally, charged and neutral grains; respectively, each normalized by the Jeans frequency $\omega_{J}$ for our low-frequency fluctuation analyses on the astrophysical scale.

We apply the standard methodology of multiscale techniques [1-2,5,16-17] over the normalized coupled set of Eqs. (12)-(24) to study the gravito-electrostatic fluctuations under weak nonlinearity approximation. Thus, the independent variables with all usual notations are stretched in to a new space defined by the $X=\epsilon^{1 / 2}(\xi-\mu \tau)$ and $T=\epsilon^{3 / 2} \tau$. In the new space, the differential operators get transformed as $\partial / \partial \xi=\epsilon^{1 / 2} \partial / \partial X$, and $\partial^{2} / \partial \xi^{2}=\in \partial^{2} / \partial X^{2}$, where $\mu$ is the normalized (by $C_{s s}$ ) phase velocity of the fluctuations and $\in$ is a minor parameter characterizing the balanced strength of nonlinearity and dispersion [1-2,5,27-28]. Accordingly, the relevant physical parameters appearing in Eqs. (12)-(24) are now expanded nonlinearly (in $\in$-powers) around the respective equilibrium values (as per the Jeans equilibrium assumption) under weak nonlinearity limit $\left(<3^{\text {rd }}\right.$ order $)$ as follows,

$$
\left(\begin{array}{l}
N_{e} \\
N_{i} \\
N_{d n} \\
N_{d c} \\
M_{e} \\
M_{i} \\
M_{d n} \\
M_{d c} \\
\Phi \\
\Psi \\
Q_{d}
\end{array}\right)=\left(\begin{array}{l}
1 \\
1 \\
1 \\
1 \\
0 \\
0 \\
0 \\
0 \\
0 \\
0 \\
0
\end{array}\right)+\in\left(\begin{array}{l}
N_{e 1} \\
N_{i 1} \\
N_{d n 1} \\
N_{d c 1} \\
M_{e 1} \\
M_{i 1} \\
M_{d n 1} \\
M_{d c 1} \\
\Phi_{1} \\
\Psi_{1} \\
Q_{d 1}
\end{array}\right)+\epsilon^{2}\left(\begin{array}{l}
N_{e 2} \\
N_{i 2} \\
N_{d n 2} \\
N_{d c 2} \\
M_{e 2} \\
M_{i 2} \\
M_{d n 2} \\
M_{d c 2} \\
\Phi_{2} \\
\Psi_{2} \\
Q_{d 2}
\end{array}\right)+\cdots
$$

We now substitute Eq. (25) in Eqs. (12)-(24) for order-by-order analyses in presence of weak nonlinearity so that higher-order perturbations may be neglected. Equating the terms in various powers of $\in$ on the fluctuations from both sides of Eq. (12), one gets

$$
\in^{1}: N_{e 1}=\Phi_{1}+\left(\frac{m_{e}}{m_{i}}\right) M_{e o}^{2} e^{\left(-2 \Phi_{o}\right)} \Phi_{1}
$$




$$
\epsilon^{2}: N_{e 2}=\Phi_{2}+\left(\frac{m_{e}}{m_{i}}\right) M_{e o}^{2} e^{\left(-2 \Phi_{o}\right)} \Phi_{2}
$$

and so on.

The order-by-order analysis in various powers of $\in$ from Eq. (13) similarly yields

$$
\begin{aligned}
& \epsilon^{1}: N_{i 1}=-\Phi_{1}-\left(\frac{m_{i}}{m_{d}}\right) M_{i o}^{2} e^{\left(2 \Phi_{o}\right)} \Phi_{1}, \\
& \epsilon^{2}: N_{i 2}=-\Phi_{2}-\left(\frac{m_{i}}{m_{d}}\right) M_{i o}^{2} e^{\left(2 \Phi_{o}\right)} \Phi_{2},
\end{aligned}
$$

and so on.

Similarly, equating the terms in various powers of $\in$ from both sides of Eq. (14), one gets

$$
\begin{gathered}
\epsilon^{3 / 2}: \frac{\partial M_{e 1}}{\partial X}-\mu \frac{\partial N_{e 1}}{\partial X}=0, \\
\epsilon^{5 / 2}: \frac{\partial N_{e 1}}{\partial T}-\mu \frac{\partial N_{e 2}}{\partial X}+N_{e 1} \frac{\partial M_{e 1}}{\partial X}+\frac{\partial M_{e 2}}{\partial X}+M_{e 1} \frac{\partial N_{e 1}}{\partial X}=0, \\
\epsilon^{7 / 2}: \frac{\partial N_{e 2}}{\partial T}+N_{e 2} \frac{\partial M_{e 1}}{\partial X}+N_{e 1} \frac{\partial M_{e 2}}{\partial X}+M_{e 2} \frac{\partial N_{e 1}}{\partial X}+M_{e 1} \frac{\partial N_{e 2}}{\partial X}=0,
\end{gathered}
$$

and so on.

The order-by-order analysis in various powers of $\in$ from Eq. (15) similarly yields

$$
\begin{gathered}
\epsilon^{3 / 2}: \frac{\partial N_{e 1}}{\partial X}+\frac{\partial \Phi_{1}}{\partial X}=0 \\
\epsilon^{5 / 2}: \frac{\partial N_{e 2}}{\partial X}+N_{e 1} \frac{\partial \Phi_{1}}{\partial X}+\frac{\partial \Phi_{2}}{\partial X}=0 \\
\epsilon^{7 / 2}: N_{e 2} \frac{\partial \Phi_{1}}{\partial X}+N_{e 1} \frac{\partial \Phi_{2}}{\partial X}=0
\end{gathered}
$$

and so forth.

Similarly, equating the terms in various powers of $\in$ from both sides of Eq. (16), one gets

$$
\begin{gathered}
\epsilon^{3 / 2}: \frac{\partial M_{i 1}}{\partial X}-\mu \frac{\partial N_{i 1}}{\partial X}=0, \\
\epsilon^{5 / 2}: \frac{\partial N_{i 1}}{\partial T}-\mu \frac{\partial N_{i 2}}{\partial X}+N_{i 1} \frac{\partial M_{i 1}}{\partial X}+\frac{\partial M_{i 2}}{\partial X}+M_{i 1} \frac{\partial N_{i 1}}{\partial X}=0, \\
\epsilon^{7 / 2}: \frac{\partial N_{i 2}}{\partial T}+N_{i 2} \frac{\partial M_{i 1}}{\partial X}+N_{i 1} \frac{\partial M_{i 2}}{\partial X}+M_{i 2} \frac{\partial N_{i 1}}{\partial X}+M_{i 1} \frac{\partial N_{i 2}}{\partial X}=0,
\end{gathered}
$$

and so on.

The order-by-order analysis in various powers of $\in$ from Eq. (17), similarly, yields

$$
\begin{gathered}
\epsilon^{3 / 2}: \frac{\partial N_{i 1}}{\partial X}+\frac{\partial \Phi_{1}}{\partial X}=0, \\
\epsilon^{5 / 2}: \frac{\partial N_{i 2}}{\partial X}+N_{i 1} \frac{\partial \Phi_{1}}{\partial X}+\frac{\partial \Phi_{2}}{\partial X}=0, \\
\epsilon^{7 / 2}: N_{i 2} \frac{\partial \Phi_{1}}{\partial X}+N_{i 1} \frac{\partial \Phi_{2}}{\partial X}=0,
\end{gathered}
$$

and so on. 
Likewise, equating the terms in various powers of $\in$ from both sides of Eq. (18), one gets

$$
\begin{gathered}
\epsilon^{3 / 2}: \frac{\partial M_{d n 1}}{\partial X}-\mu \frac{\partial N_{d n 1}}{\partial X}=0 \\
\epsilon^{5 / 2}: \frac{\partial N_{d n 1}}{\partial T}-\mu \frac{\partial N_{d n 2}}{\partial X}+N_{d n 1} \frac{\partial M_{d n 1}}{\partial X}+\frac{\partial M_{d n 2}}{\partial X}+M_{d n 1} \frac{\partial N_{d n 1}}{\partial X}=0 \\
\epsilon^{7 / 2}: \frac{\partial N_{d n 2}}{\partial T}+N_{d n 2} \frac{\partial M_{d n 1}}{\partial X}+N_{d n 1} \frac{\partial M_{d n 2}}{\partial X}+M_{d n 2} \frac{\partial N_{d n 1}}{\partial X}+M_{d n 1} \frac{\partial N_{d n 2}}{\partial X}=0
\end{gathered}
$$

and so on.

The order-by-order analysis in various powers of $\in$ from Eq. (19), similarly, yields

$$
\begin{gathered}
\epsilon^{3 / 2}: \frac{1}{n_{d n o}} \frac{\partial N_{d n 1}}{\partial X}-\mu \frac{\partial M_{d n 1}}{\partial X}+\left(\frac{m_{d n}}{n_{d c o} e}\right) \frac{\partial \Psi_{1}}{\partial X}=0, \\
\epsilon^{5 / 2}: \frac{\partial M_{d n 1}}{\partial T}-\mu \frac{\partial M_{d n 2}}{\partial X}+M_{d n 1} \frac{\partial M_{d n 1}}{\partial X}-\mu N_{d n 1} \frac{\partial M_{d n 1}}{\partial X} \\
\quad+\frac{1}{n_{d n o}} \frac{\partial N_{d n 2}}{\partial X}+\left(\frac{m_{d n}}{n_{d n o} e}\right) N_{d n 1} \frac{\partial \Psi_{1}}{\partial X}+\left(\frac{m_{d n}}{n_{d n o} e}\right) \frac{\partial \Psi_{2}}{\partial X}=0,
\end{gathered}
$$

and so on.

Alike, equating the terms in various powers of $\in$ from both sides of Eq. (20), one gets

$$
\begin{gathered}
\epsilon^{3 / 2}: \frac{\partial M_{d c 1}}{\partial X}-\mu \frac{\partial N_{d c 1}}{\partial X}=0, \\
\epsilon^{5 / 2}: \frac{\partial N_{d c 1}}{\partial T}-\mu \frac{\partial N_{d c 2}}{\partial X}+N_{d c 1} \frac{\partial M_{d c 1}}{\partial X}+\frac{\partial M_{d c 2}}{\partial X}+M_{d c 1} \frac{\partial N_{d c 1}}{\partial X}=0, \\
\epsilon^{7 / 2}: \frac{\partial N_{d c 2}}{\partial T}+N_{d c 2} \frac{\partial M_{d c 1}}{\partial X}+N_{d c 1} \frac{\partial M_{d c 2}}{\partial X}+M_{d c 2} \frac{\partial N_{d c 1}}{\partial X}+M_{d c 1} \frac{\partial N_{d c 2}}{\partial X}=0,
\end{gathered}
$$

and so on

The order-by-order analysis in various powers of $\in$ from Eq. (21) similarly yields

$$
\begin{aligned}
& \epsilon^{3 / 2}: \frac{1}{n_{d c o}} \frac{\partial N_{d c 1}}{\partial X}-\mu \frac{\partial M_{d c 1}}{\partial X}+\left(\frac{m_{d c}}{n_{d c o} e}\right) \frac{\partial \Psi_{1}}{\partial X}=0, \\
& \epsilon^{5 / 2}: \frac{\partial M_{d c 1}}{\partial T}-\mu \frac{\partial M_{d c 2}}{\partial X}+M_{d c 1} \frac{\partial M_{d c 1}}{\partial X}-\mu N_{d c 1} \frac{\partial M_{d c 1}}{\partial X} \\
& +\frac{1}{n_{d c o}} \frac{\partial N_{d c 2}}{\partial X}+\left(\frac{m_{d c}}{n_{d c o} e}\right) N_{d c 1} \frac{\partial \Psi_{1}}{\partial X}+\left(\frac{m_{d c}}{n_{d c o} e}\right) \frac{\partial \Psi_{2}}{\partial X}+\left(\frac{q_{d o}}{n_{d c o}}\right) Q_{d 1} \frac{\partial \Phi_{1}}{\partial X}=0, \\
& \epsilon^{7 / 2}: \frac{\partial M_{d c 2}}{\partial T}+M_{d c 2} \frac{\partial M_{d c 1}}{\partial X}+M_{d c 1} \frac{\partial M_{d c 2}}{\partial X}+N_{d c 1} \frac{\partial M_{d c 1}}{\partial T}-\mu N_{d c 1} \frac{\partial M_{d c 2}}{\partial X} \\
& +N_{d c 1} M_{d c 1} \frac{\partial M_{d c 1}}{\partial X}-\mu N_{d c 2} \frac{\partial M_{d c 1}}{\partial X}+\left(\frac{m_{d c}}{n_{d c o} e}\right) N_{d c 2} \frac{\partial \Psi_{1}}{\partial X}+\left(\frac{m_{d c}}{n_{d c o} e}\right) N_{d c 1} \frac{\partial \Psi_{2}}{\partial X} \\
& +\left(\frac{q_{d o}}{n_{d c o}}\right) Q_{d 2} \frac{\partial \Phi_{1}}{\partial X}+\left(\frac{q_{d o}}{n_{d c o}}\right) Q_{d 1} N_{d c 1} \frac{\partial \Phi_{1}}{\partial X}+\left(\frac{q_{d o}}{n_{d c o}}\right) Q_{d 1} \frac{\partial \Phi_{2}}{\partial X}=0, \\
& \epsilon^{9 / 2}: M_{d c 2} \frac{\partial M_{d c}}{\partial X}+N_{d c 1} \frac{\partial M_{d c 2}}{\partial T}+N_{d c 1} M_{d c 2} \frac{\partial M_{d c 1}}{\partial X}+N_{d c 1} M_{d c 1} \frac{\partial M_{d c 2}}{\partial X}+N_{d c 2} \frac{\partial M_{d c 1}}{\partial T} \\
& -\mu N_{d c 2} \frac{\partial M_{d c 2}}{\partial X}+N_{d c 2} M_{d c 1} \frac{\partial M_{d c 1}}{\partial X}+\left(\frac{m_{d c}}{n_{d c o} e}\right) N_{d c 2} \frac{\partial \Psi_{2}}{\partial X}+\left(\frac{q_{d o}}{n_{d c o}}\right) Q_{d 2} N_{d c 1} \frac{\partial \Phi_{1}}{\partial X} \\
& +\left(\frac{q_{d o}}{n_{d c o}}\right) Q_{d 1} N_{d c 2} \frac{\partial \Phi_{1}}{\partial X}+\left(\frac{q_{d o}}{n_{d c o}}\right) Q_{d 2} \frac{\partial \Phi_{2}}{\partial X}+\left(\frac{q_{d o}}{n_{d c o}}\right) Q_{d 1} N_{d c 1} \frac{\partial \Phi_{2}}{\partial X}=0, \\
& \in^{11 / 2}: N_{d c 1} M_{d c 2} \frac{\partial M_{d c}}{\partial X}+N_{d c 2} \frac{\partial M_{d c 1}}{\partial T}+N_{d c 2} M_{d c 2} \frac{\partial M_{d c 1}}{\partial X}+N_{d c 2} M_{d c 1} \frac{\partial M_{d c 2}}{\partial X} \\
& +\left(\frac{q_{d o}}{n_{d c o}}\right) Q_{d 2} N_{d c 2} \frac{\partial \Phi_{1}}{\partial X}++\left(\frac{q_{d o}}{n_{d c o}}\right) Q_{d 2} N_{d c 1} \frac{\partial \Phi_{2}}{\partial X}+\left(\frac{q_{d o}}{n_{d c o}}\right) Q_{d 1} N_{d c 2} \frac{\partial \Phi_{2}}{\partial X}=0,
\end{aligned}
$$




$$
\in^{14 / 2}: N_{d c 2} M_{d c 2} \frac{\partial M_{d c 2}}{\partial X}+\left(\frac{q_{d o}}{n_{d c o}}\right) Q_{d 2} N_{d c 2} \frac{\partial \Phi_{2}}{\partial X}=0
$$

and so on.

Similarly, equating the terms in various powers of $\in$ from both sides of Eq. (22), one gets

$$
\begin{gathered}
\in^{1}: \frac{e}{m_{d c}^{2} n_{d o} G}\left(e n_{e o} N_{e 1}-e n_{i o} N_{i 1}+q_{d o} n_{d c o} Q_{d 1}\right)=0, \\
\epsilon^{2}: \frac{\partial^{2} \Phi_{1}}{\partial X^{2}}=\frac{e}{m_{d c}^{2} n_{d o} G}\left(e n_{e o} N_{e 2}-e n_{i o} N_{i 2}+q_{d o} n_{d c o} Q_{d 2}+q_{d o} n_{d c o} Q_{d 1} N_{d c 1}\right), \\
\epsilon^{3}: \frac{\partial^{2} \Phi_{2}}{\partial X^{2}}=\frac{e}{m_{d c}^{2} n_{d o} G}\left(q_{d o} n_{d c o} Q_{d 2} N_{d c 1}+q_{d o} n_{d c o} Q_{d 1} N_{d c 2}\right), \\
\in^{4}: q_{d o} n_{d c o} Q_{d 2} N_{d c 2}=0,
\end{gathered}
$$

and so on.

The order-by-order analysis in various powers of $\in$ from Eq. (23) similarly yields

$$
\begin{gathered}
\in^{1}: \frac{e}{m_{d n} n_{d o}}\left\{n_{d n o} N_{d n 1}+n_{d c o} N_{d c 1}+n_{e o}\left(\frac{m_{e}}{m_{d}}\right) N_{e 1}+n_{i o}\left(\frac{m_{i}}{m_{d}}\right) N_{i 1}\right\}=0, \\
\in^{2}: \frac{\partial^{2} \Psi_{1}}{\partial X^{2}}=\frac{e}{m_{d n} n_{d o}}\left\{n_{d n o} N_{d n 2}+n_{d c o} N_{d c 2}+n_{e o}\left(\frac{m_{e}}{m_{d}}\right) N_{e 2}+n_{i o}\left(\frac{m_{i}}{m_{d}}\right) N_{i 2}\right\}, \\
\in^{3}: \frac{\partial^{2} \Psi_{2}}{\partial X^{2}}=0,
\end{gathered}
$$

and so forth.

Similarly, equating the terms in various powers of $\in$ from both sides of Eq. (24), one gets

$$
\begin{gathered}
\epsilon^{1}: \frac{e}{q_{d o}}\left(\frac{n_{e o}}{n_{d o}}\right) F_{e d}\left\{N_{e 1}-\left(\frac{n_{i o}}{n_{e o}}\right) \frac{F_{i d}}{F_{e d}} N_{i 1}\right\}=0 \\
\epsilon^{2}: \frac{e}{q_{d o}}\left(\frac{n_{e o}}{n_{d o}}\right) F_{e d}\left\{N_{e 2}-\left(\frac{n_{i o}}{n_{e o}}\right) \frac{F_{i d}}{F_{e d}} N_{i 2}\right\}=0 \\
\epsilon^{3 / 2}:-\mu \frac{\partial Q_{d 1}}{\partial X}=0 \\
\epsilon^{5 / 2}: \frac{\partial Q_{d 1}}{\partial T}-\mu \frac{\partial Q_{d 2}}{\partial X}+M_{d c 1} \frac{\partial Q_{d 1}}{\partial X}=0 \\
\epsilon^{7 / 2}: \frac{\partial Q_{d 2}}{\partial T}+M_{d c 2} \frac{\partial Q_{d 1}}{\partial X}+M_{d c 1} \frac{\partial Q_{d 1}}{\partial X}=0 \\
\epsilon^{9 / 2}: M_{d c 2} \frac{\partial Q_{d 2}}{\partial X}=0
\end{gathered}
$$

and so on. 


\section{IV.1. Derivation of electrostatic d-KdV equation}

After simplifying Eq. (57), by systematically eliminating the second-order perturbed quantities using various Eqs. (26)-(68), we get the following $d$-KdV equation describing the nonlinear electrostatic fluctuations (in terms of $\Phi_{1}$ ), expressed as,

$$
\frac{\partial \Phi_{1}}{\partial T}+A_{1} \Phi_{1} \frac{\partial \Phi_{1}}{\partial X}+B_{1} \frac{\partial^{3} \Phi_{1}}{\partial X^{3}}=S_{o}
$$

where

$$
S_{o}=-C_{1} \Phi_{1}^{3} \frac{\partial \Phi_{1}}{\partial X}-D_{1} \Phi_{1}^{2} \frac{\partial \Phi_{1}}{\partial X}
$$

This is the derived $d-\mathrm{KdV}$ equation with a self-consistent source $S_{o}$, which comes mainly due to the consideration of the lowest-order inertia of the thermal species. The various coefficients are

$$
\begin{gathered}
A_{1}=\frac{-\mu}{\left[n_{i o}\left\{1+\left(\frac{m_{i}}{m_{d}}\right) M_{i o}^{2}\right\}+n_{e o}\left\{1+\left(\frac{m_{e}}{m_{i}}\right) M_{e o}^{2}\right\}\right]} \\
\times\left[\frac{n_{e o}\left\{1+\left(\frac{m_{e}}{m_{i}}\right) M_{e o}^{2}\right\}^{2}}{\left\{2+\left(\frac{m_{e}}{m_{i}}\right) M_{e o}^{2}\right\}}+\frac{\left.n_{i o}\left\{1+\left(\frac{m_{i}}{m_{d}}\right) M_{i o}^{2}\right\}^{2}\right]}{\left(\frac{m_{i}}{m_{d}}\right) M_{i o}^{2}}\right], \\
B_{1}=-\frac{m_{d}^{2} n_{d o} G \mu}{e^{2}\left[n_{i o}\left\{1+\left(\frac{m_{i}}{m_{d}}\right) M_{i o}^{2}\right\}+n_{e o}\left\{1+\left(\frac{m_{e}}{m_{i}}\right) M_{e o}^{2}\right\}\right]}, \\
C_{1}=\frac{e\left\{1+\left(\frac{m_{i}}{m_{d}}\right) M_{i o}^{2}\right\}}{\mu n_{d c o}^{2}\left\{2+\left(\frac{m_{e}}{m_{i}}\right) M_{e o}^{2}\right\}}\left[n_{i o}\left\{1+\left(\frac{m_{i}}{m_{d}}\right) M_{i o}^{2}\right\}+n_{e o}\left\{1+\left(\frac{m_{e}}{m_{i}}\right) M_{e o}^{2}\right\}\right]
\end{gathered}
$$

and

$$
D_{1}=-\frac{e}{\mu n_{d c o}^{2}}\left[n_{i o}\left\{1+\left(\frac{m_{i}}{m_{d}}\right) M_{i o}^{2}\right\}+n_{e o}\left\{1+\left(\frac{m_{e}}{m_{i}}\right) M_{e o}^{2}\right\}\right] .
$$

\section{IV.2. Derivation of self-gravitational KdV equation}

By simplifying Eq. (61), with the methodological elimination procedure of the second-order perturbed quantities using relations from Eqs. (26)-(68), we obtain the following KdV equation depicting nonlinear self-gravitational fluctuations (in terms of $\Psi_{1}$ ), as,

$$
\frac{\partial \Psi_{1}}{\partial T}+A_{2} \Psi_{1} \frac{\partial \Psi_{1}}{\partial X}+B_{2} \frac{\partial^{3} \Psi_{1}}{\partial X^{3}}=0
$$

This is the derived $\mathrm{KdV}$ equation with the coefficients $A_{2}=Z_{2} / Z_{1}$ and $B_{2}=\left(-1 / Z_{1}\right)$, where,

$$
Z_{1}=\frac{2 \mu n_{d n o}^{2}}{n_{d o}\left(1-\mu^{2} n_{d n o}\right)^{2}}-\frac{1}{\mu m_{d} n_{d o}}\left\{\begin{array}{l}
\frac{n_{d n o}}{\left(\mu^{2} n_{d n o}-1\right)} \\
+\frac{n_{d c o}}{\left(\mu^{2} n_{d c o}-1\right)}
\end{array}\right\}\left[\begin{array}{c}
\frac{m_{e}}{\left\{\left(\frac{m_{e}}{m_{d}}\right)+\left(\frac{m_{i}}{m_{d}}\right) \frac{F_{e d}}{F_{i d}}\right\}} \\
+\frac{m_{i}}{\left\{\left(\frac{m_{i}}{m_{d}}\right)+\left(\frac{m_{e}}{m_{d}}\right) \frac{F_{i d}}{F_{e d}}\right\}}
\end{array}\right],
$$




$$
\begin{aligned}
Z_{2}= & \frac{2}{n_{d o} e}\left\{\begin{array}{l}
\frac{n_{d n o}}{\left(\mu^{2} n_{d n o}-1\right)} \\
+\frac{n_{d c o}}{\left(\mu^{2} n_{d c o}-1\right)}
\end{array}\right\}^{2}\left[\frac{m_{e}}{n_{e o}\left\{\left(\frac{m_{e}}{m_{d}}\right)+\left(\frac{m_{i}}{m_{d}}\right) \frac{F_{e d}}{F_{i d}}\right\}^{2}}+\frac{m_{i}}{n_{i o}\left\{\left(\frac{m_{i}}{m_{d}}\right)+\left(\frac{m_{e}}{m_{d}}\right) \frac{F_{i d}}{F_{e d}}\right\}^{2}}\right] \\
& +\frac{m_{d} n_{d n o}}{n_{d o} e\left(\mu^{2} n_{d n o}-1\right)^{2}}\left\{\frac{2 n_{d n o} \mu^{2}}{\left(\mu^{2} n_{d n o}-1\right)}+1\right\} .
\end{aligned}
$$

\section{IV.3. Stationary evolution equations}

We are interested in simplified time-stationary (steady-state) solutions to study the basic characteristics of the gravito-electrostatic fluctuation dynamics of the DMC [15-16]. Although this technique is well-known to readers, we want to show it for learners. Thus, Eqs. (69) and (75) are transformed into ordinary differential equations (ODEs) with the Galilean type of transformation [15-16] $\rho=X-T$ (co-moving coordinate) so that the differential operator transformation becomes $\partial / \partial X=\partial / \partial \rho$ and $\partial / \partial T=-\partial / \partial \rho$. Consequently, their stationary forms, respectively, are presented as follows,

$$
\frac{\partial \Phi_{1}}{\partial \rho}-A_{1} \Phi_{1} \frac{\partial \Phi_{1}}{\partial \rho}-B_{1} \frac{\partial^{3} \Phi_{1}}{\partial \rho^{3}}=S_{o}
$$

where

and

$$
S_{o}=C_{1} \Phi_{1}^{3} \frac{\partial \Phi_{1}}{\partial \rho}+D_{1} \Phi_{1}^{2} \frac{\partial \Phi_{1}}{\partial \rho}
$$

$$
\frac{\partial \Psi_{1}}{\partial \rho}-A_{2} \Psi_{1} \frac{\partial \Psi_{1}}{\partial \rho}-B_{2} \frac{\partial^{3} \Psi_{1}}{\partial \rho^{3}}=0
$$

Thus, the steady-state structures of the cloud fluctuations are governed by the nonlinear ordinary differential equations (78) and (79), are likely to grow as solitary spectral patterns.

\section{RESULTS AND DISCUSSIONS}

A simplified theoretical model to describe the properties of nonlinear gravito-electrostatic fluctuation dynamics in the cloud in the small amplitude limit (weak nonlinearity) is proposed with all the possible realistic dynamical agencies taken into account for the first time. The lowest-order eigenmode evolutions are expressible by the KdV-type equations ( $d-\mathrm{KdV}$ for the electrostatic, and $\mathrm{KdV}$ for the self-gravitational fluctuations) obtained by multiscale analyses, where, higher-order perturbations are neglected due to weak nonlinearity. They are simulated numerically as initial value problems in a time-stationary configuration by the fourth-order Runge-Kutta method under some judicious multi-parameter variation dependent on the cloud. The numerical results are shown in two subsections, one for the electrostatic $d-\mathrm{KdV}$ equation, and the other for the self-gravitational KdV equation.

\section{V.1. Electrostatic fluctuations}

The nonlinear electrostatic fluctuation dynamics in presence of the inertia-modified Boltzmann distributed thermal species, non-adiabatic dust-charge variation and all the possible collisional processes is collectively governed by the $d-\mathrm{KdV}$ equation (Eq. (69)). It contains a selfconsistent nonlinear driving source term arising due to the weak inertial effect, equilibrium flow of thermal species and equilibrium charged dust distribution (as clear from Eq. (70) in association with Eqs. (73)-(74)). To see the graphical nature of the patterns and internal microphysics of the fluctuations, the $d-\mathrm{KdV}$ equation is simulated numerically. The obtained results are shown in Figs. 
1-4. All the profiles obtained by suitable choice different judicious values of plasma parameters and initial inputs [12-17] are elaborately discussed below.

Figure 1 depicts the profiles of the lowest-order perturbed electrostatic (a) potential, (b) field, (c) potential curvature, and (d) phase portrait. Various lines link to Case (1): $m_{d}=1.078 \times$ $10^{-11} \mathrm{~kg}$ (blue line), Case (2): $m_{d}=1.079 \times 10^{-11} \mathrm{~kg}$ (red line), Case (3): $m_{d}=1.080 \times 10^{-11} \mathrm{~kg}$ (green line), and Case (4): $m_{d}=1.081 \times 10^{-11} \mathrm{~kg}$ (black line), respectively. Different input initial values used are $(\Phi)_{i}=0.00,\left(\Phi_{\rho}\right)_{i}=1.00 \times 10^{-8}$, and $\left(\Phi_{\rho \rho}\right)_{i}=1.07 \times 10^{-6}$. The other parameters kept fixed are $M_{e o}=1.00 \times 10^{-2}, M_{i o}=1.00 \times 10^{-3}, m_{e}=9.109 \times 10^{-31} \mathrm{~kg}$, $m_{i}=1.673 \times 10^{-27} \mathrm{~kg}, n_{e o}=2.00 \times 10^{8} \mathrm{~m}^{-3}, n_{i o}=3.15 \times 10^{9} \mathrm{~m}^{-3}, n_{\text {dno }}=5.00 \times 10^{6} \mathrm{~m}^{-3}$, $n_{d c o}=5.50 \times 10^{5} \mathrm{~m}^{-3}$, and $\mu=1.00$. The potential fluctuation (Fig. 1a) displays driven (global average amplitude increasing) damped oscillatory (local oscillation amplitude decreasing) shocklike eigenmodes, which are compressive in nature. The damped oscillatory shock-like nature is due to the dissipative non-adiabatic dust-charge variation. The oscillatory behavior alone is because of the continuous compressive and rarefactive racing of the dust and thermal species amid the periodic interplay of gravito-electrostatic coupling. It is found that with slight increase in grain-mass, the global amplitude increases after $\rho>6.5 \lambda_{J}$ with more damping effect in local oscillations. It may be due basically to the fact the self-gravitational attraction increases with the grain-mass, which decreases the electrostatic repulsive action between the like Columbic particles with distance. This may increase the damping effect on the exterior scale relative to the centre. The corresponding periodic-type variation in the field (Fig. 1b), curvature (Fig. 1c) and geometrical patterns (Fig. 1d) strengthen the graphical interpretation of the associated dissipative dynamics due to the grain-charge fluctuations. The open structures in the phase show signatures of the dissipative saturation mechanism in presence of weak thermal inertia and nonlinearity.

Figure 2 shows the same as Fig. 1, but under different conditions with fixed $m_{d}=1.079 \times$ $10^{-11} \mathrm{~kg}$. Various lines correspond to Case (1): $n_{d n o}=5.00 \times 10^{6} \mathrm{~m}^{-3}$ (blue line), Case (2): $n_{d n o}=5.02 \times 10^{6} \mathrm{~m}^{-3}$ (red line), Case (3): $n_{d n o}=5.04 \times 10^{6} \mathrm{~m}^{-3}$ (green line), and Case (4): $n_{\text {dno }}=5.06 \times 10^{6} \mathrm{~m}^{-3}$ (black line), respectively.

Figure 3 portrays the same as Figs. 1, but with $m_{d}=8.00 \times 10^{-11} \mathrm{~kg}$. Various lines correspond to Case (1): $n_{d c o}=1.50 \times 10^{5} \mathrm{~m}^{-3}$ (blue line), Case (2): $n_{d c o}=3.50 \times 10^{5} \mathrm{~m}^{-3}$ (red line), Case (3): $n_{d c o}=5.50 \times 10^{5} \mathrm{~m}^{-3}$ (green line), and Case (4): $n_{d c o}=7.50 \times 10^{5}$ $\mathrm{m}^{-3}$ (black line), respectively. It is clear from the potential profile (Fig. 3a) that with relatively higher grain-mass, the oscillatory shock-like nature disappears, and two-tail compressive solitons (extended) evolve. This is in good agreement with the observations made by others in different situations [2,5,8,28-30]. The driving effect of the nonlinear source is now negligible as its strength is weakened by the self-gravity. Moreover, the amplitude of two-tail soliton increases with increase in equilibrium value of the charged grain density, and vice versa. With the increase in equilibrium charged grain density, the electrostatic repulsive force between the grains increases against the self-gravitational attractive force. This assists a slight increase of the wave amplitude. The corresponding field-fluctuation (Fig. 3b) evolves as soliton-antisoliton mixtures. The perturbed deviation from quasi-neutrality (Fig. 3c) appears as rarefactive solitary patterns (extended). In addition, the geometrical trajectories (Fig. 3d) are closed orbits characterizing a conservative nature of the system.

Figure 4 displays the same as Fig. 1, but for different grain-masses showing an interesting transition from oscillatory shock (with smaller average amplitude) to soliton (with larger average 


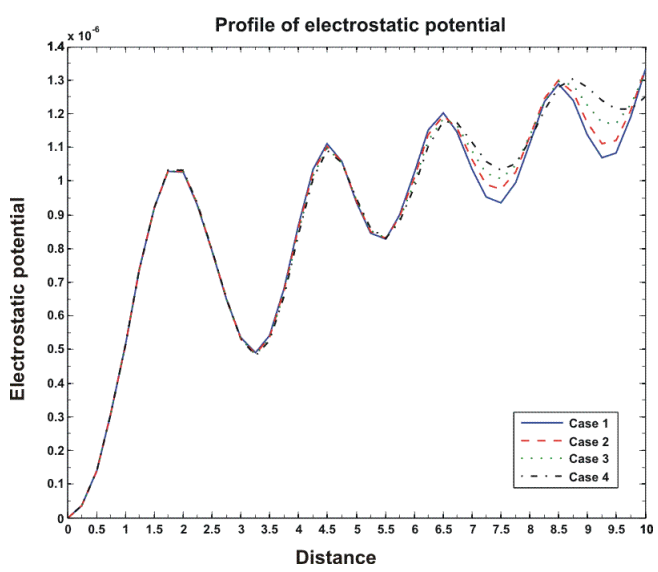

(a)

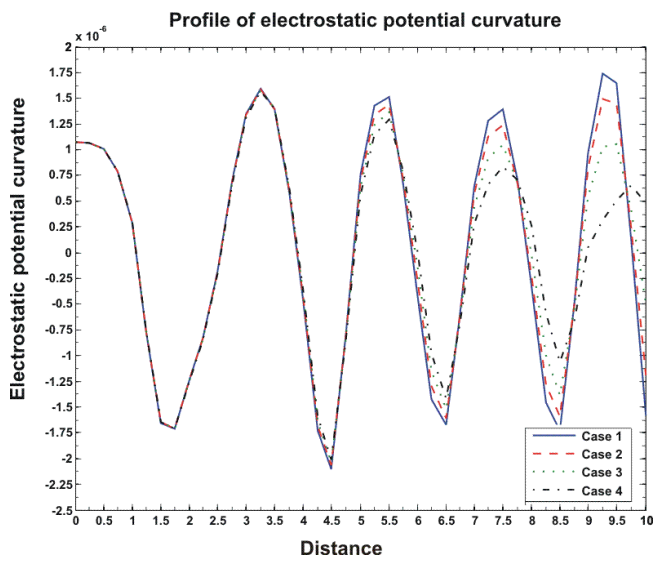

(c)

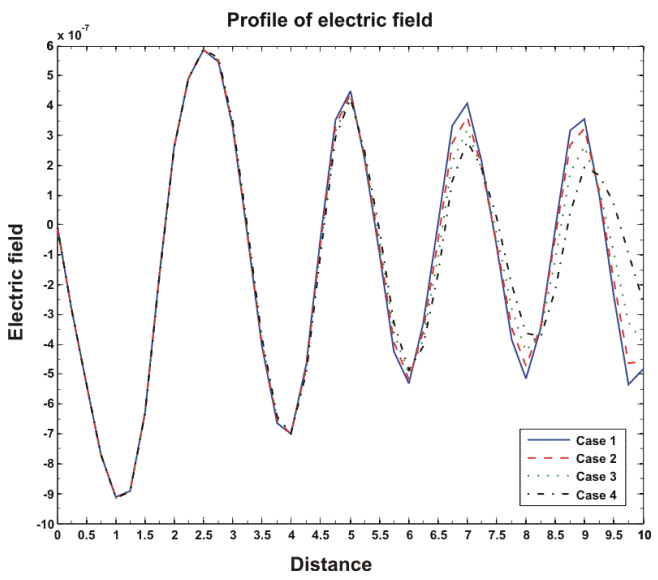

(b)

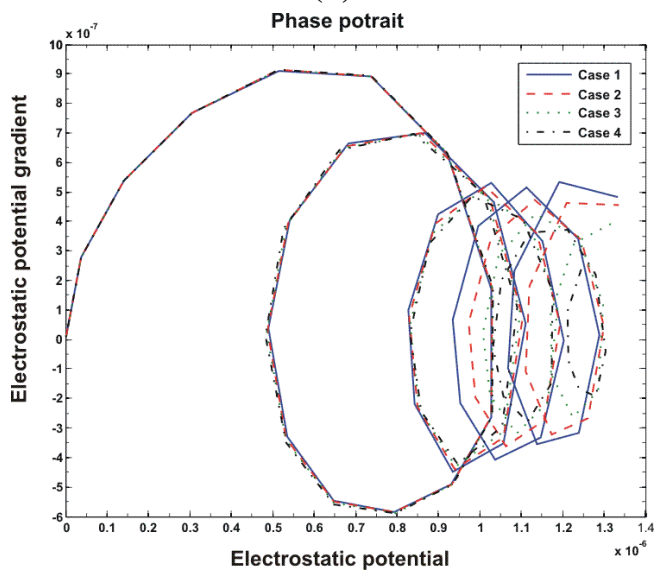

(d)

Fig. 1. Profile of the lowest-order perturbed electrostatic (a) potential, (b) field, (c) potential curvature, and (d) phase portrait. Various lines correspond to Case (1): $m_{d}=$ $1.078 \times 10^{-11} \mathrm{~kg}$ (blue line), Case (2): $m_{d}=1.079 \times 10^{-11} \mathrm{~kg}$ (red line), Case (3): $m_{d}=1.080 \times 10^{-11} \mathrm{~kg}$ (green line), and Case (4): $m_{d}=1.081 \times 10^{-11} \mathrm{~kg}$ (black line), respectively. Different fixed and input initial values used are given in the text.

amplitude). Various lines correspond to Case (1): $m_{d}=1.079 \times 10^{-11} \mathrm{~kg}$ (blue line, rescaled by multiplying with 3.00 ), Case (2): $m_{d}=3.40 \times 10^{-11} \mathrm{~kg}$ (red line, rescaled by multiplying with 1.50), Case (3): $m_{d}=5.70 \times 10^{-11} \mathrm{~kg}$ (green line, rescaled by multiplying with 1.10 ), and Case (4): $m_{d}=8.00 \times 10^{-11} \mathrm{~kg}$ (black line), respectively. It is seen that the grain-mass variation has a direct correlation with the transition from damped oscillatory shock-like eigenmode to twotail compressive soliton-like structure (Fig. 4a). For low grain-mass, it is known that collisions and non-adiabatic dust-charge variation (dissipative) dominates the self-gravitational dispersive effect. So, the dissipative process tends to balance with the wave-breaking convective force of nonlinear nature. Under suitable condition, it gives rise to the generation of damped oscillatory 


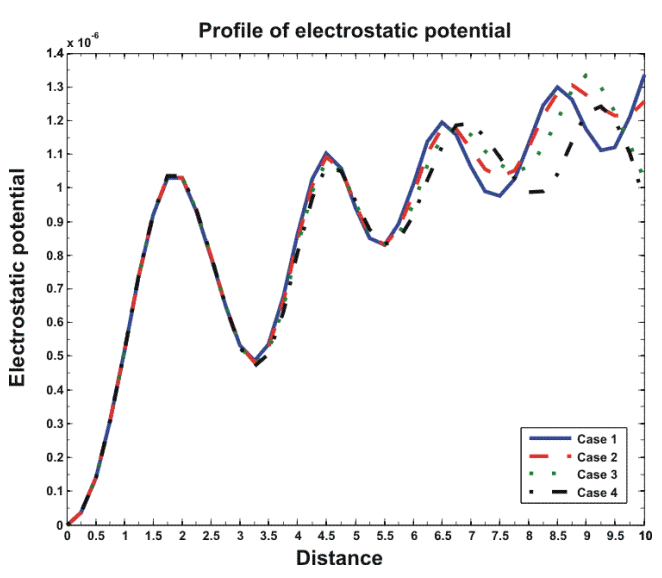

(a)

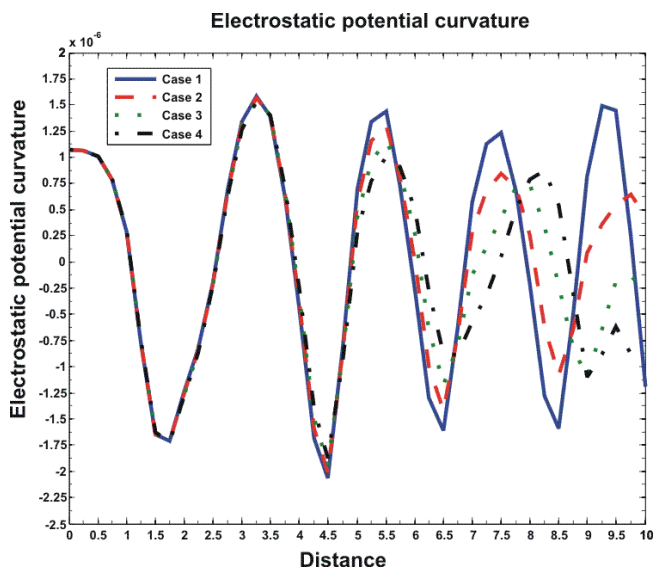

(c)

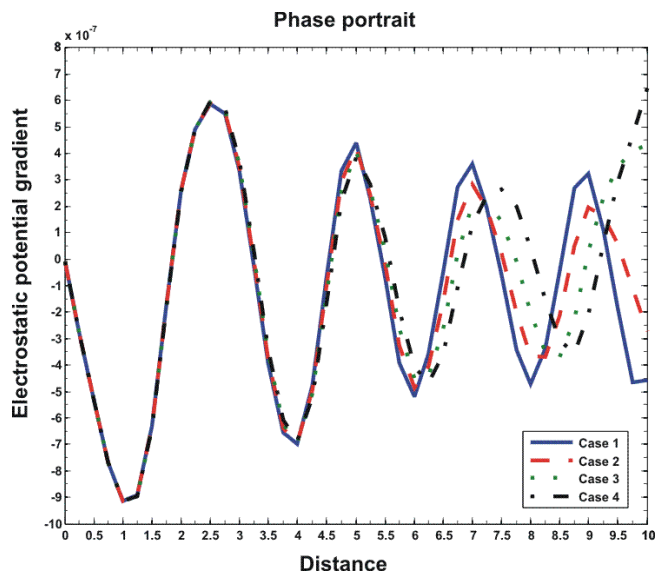

(b)

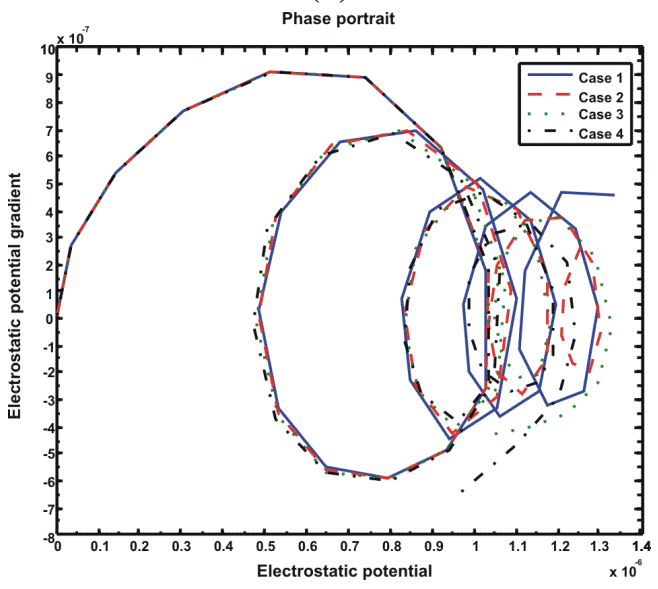

(d)

Fig. 2. Same as Fig. 1, but with $m_{d}=1.079 \times 10^{-11} \mathrm{~kg}$. Various lines correspond to Case (1): $n_{d n o}=5.00 \times 10^{6} \mathrm{~m}^{-3}$ (blue line), Case (2): $n_{d n o}=5.02 \times 10^{6} \mathrm{~m}^{-3}$ (red line), Case (3): $n_{d n o}=5.04 \times 10^{6} \mathrm{~m}^{-3}$ (green line), and Case (4): $n_{d n o}=5.06 \times 10^{6} \mathrm{~m}^{-3}$ (black line), respectively.

shock-like eigenmodes. As the mass of the grain increases, the self-gravitational dispersive effects through geometrical curvature effect become sufficient to dominate the dissipative collisional and charge-variation processes. The interplay between nonlinear (hydrodynamic origin) and dispersive (self-gravitational origin) effects leads to the growth of such saturated structures. The consistent variations in the field (Fig. 4b), curvature (Fig. 4c) and geometrical patterns (Fig. 4d) equally support this dissipative-to-conservative transition of the fluctuation saturation mechanism. The theoretical analysis for the electrostatic fluctuations allows us to summarize some other main points of astrophysical interest as follows. 


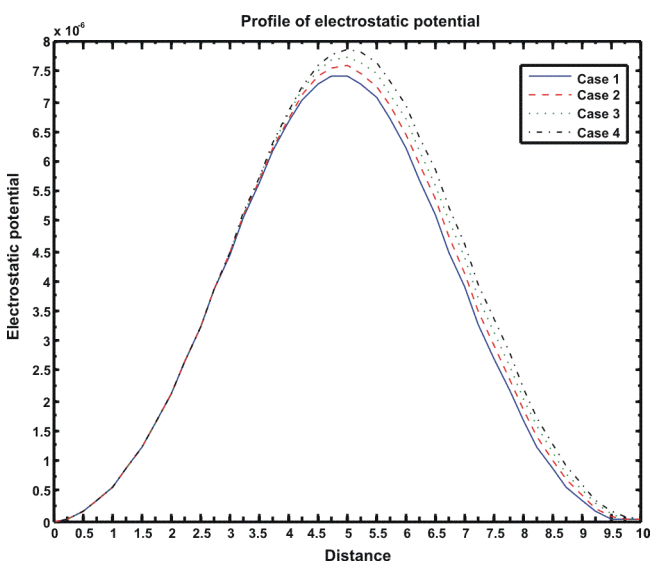

(a)

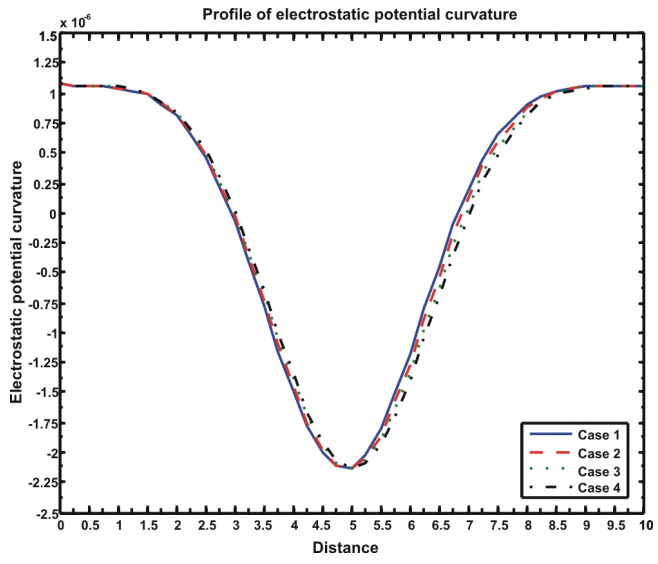

(c)

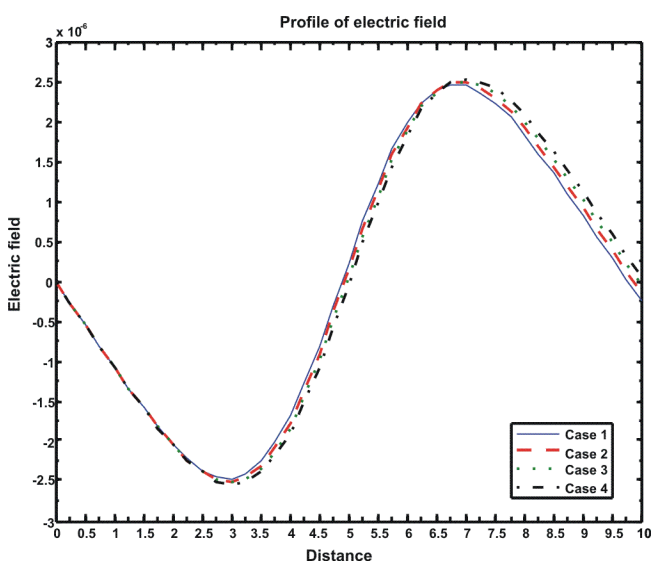

(b)

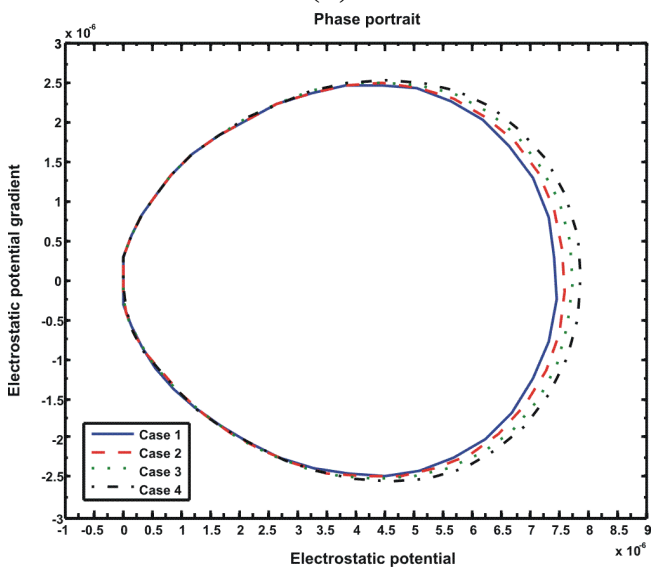

(d)

Fig. 3. Same as Fig. 1, but with $m_{d}=8.00 \times 10^{-11} \mathrm{~kg}$. Various lines correspond to Case (1): $n_{d c o}=1.50 \times 10^{5} \mathrm{~m}^{-3}$ (blue line), Case (2): $n_{d c o}=3.50 \times 10^{5} \mathrm{~m}^{-3}$ (red line), Case (3): $n_{d c o}=5.50 \times 10^{5} \mathrm{~m}^{-3}$ (green line), and Case (4): $n_{d c o}=7.50 \times 10^{5} \mathrm{~m}^{-3}$ (black line), respectively.

The potential fluctuation profiles (Figs.1a-4a) show a two-scale behavior. First, the shortscale fluctuation dynamics evolving as damped oscillator, where the oscillation amplitude decreases rapidly with distance, and finally, gets damped out nearly to zero. Second, the large-scale fluctuation dynamics evolving as shock-like structures, where the average shock amplitude increases slowly with distance relative to the centre of the total cloud mass distribution. The driven nature of the wave amplitude for the large-scale behavior is due to the self-consistent source, although its strength is small (roughly, $S_{o} \sim 3.447 \times 10^{-42}$ ) and short-scale amplitude decreases due to the decreasing tendency of the periodic gravito-electrostatic coupling strength.

For low grain-mass $\left(m_{d} \sim 1.078 \times 10^{-11} \mathrm{~kg}\right)$, the numerical value of convective coefficient $\left|A_{1}\right| \sim 2.718 \times 10^{9}$, dispersive term $\left|B_{1}\right| \sim 4.607 \times 10^{2}$ and source term $S_{o} \sim 3.447 \times 10^{-42}$. Although, the source is weak, it drives the large-scale average shock-amplitude. The strengths for 


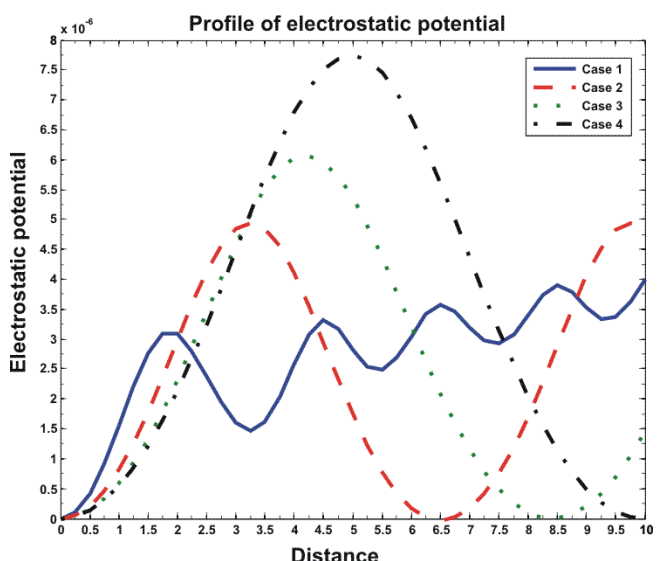

(a)

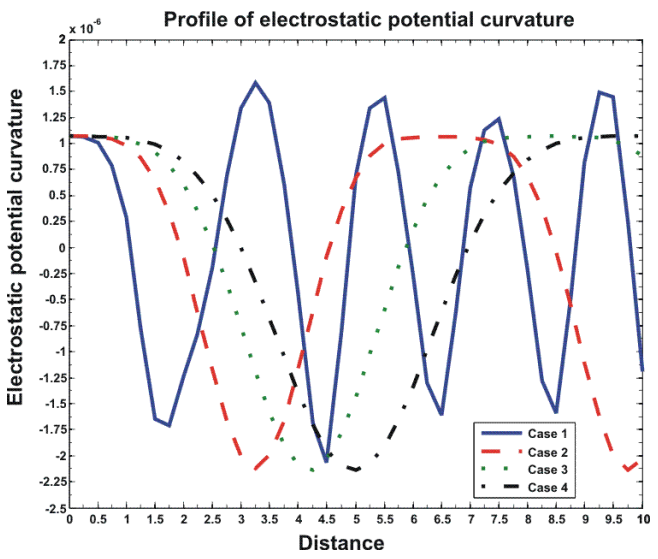

(c)

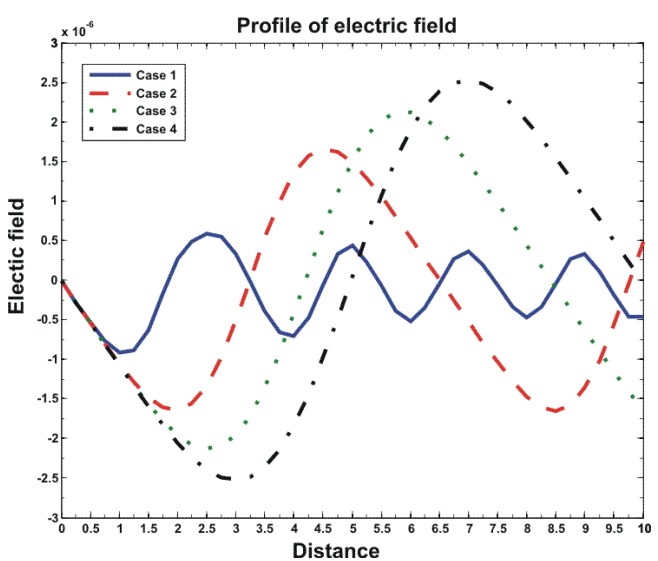

(b)

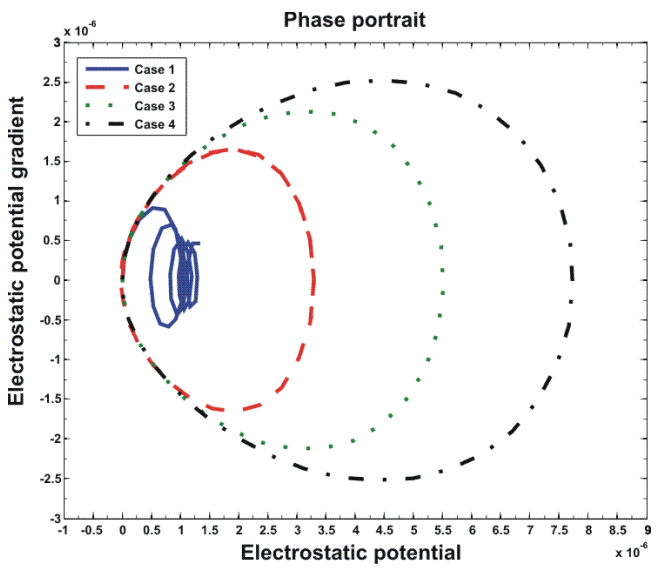

(d)

Fig. 4. Same as Fig. 1, but for different grain masses showing shock-to-soliton transition. Various lines correspond to Case (1): $m_{d}=1.079 \times 10^{-11} \mathrm{~kg}$ (blue line rescaled by multiplying with 3.00), Case (2): $m_{d}=3.40 \times 10^{-11} \mathrm{~kg}$ (red line rescaled by multiplying with 1.50), Case (3): $m_{d}=5.70 \times 10^{-11} \mathrm{~kg}$ (green line rescaled by multiplying with 1.10), and Case (4): $m_{d}=8.00 \times 10^{-11} \mathrm{~kg}$ (black line), respectively.

higher grain mass $\left(m_{d} \sim 8.00 \times 10^{-11} \mathrm{~kg}\right)$ are $\left|A_{1}\right| \sim 2.718 \times 10^{9},\left|B_{1}\right| \sim 2.538 \times 10^{4}$ and $S_{0} \sim 3.447 \times 10^{-52}$. It is clear that with higher grain-mass, the strength of the dispersive term increases tremendously $\left(\left|B_{11}\right| /\left|B_{12}\right| \sim 10^{-2}\right)$ supporting the shock-to-soliton transition. The driving nature of the eigenmode is not found for higher grain-mass fluctuation profiles due basically to the decrease in strength of the source term $\left(S_{o 1} / S_{02} \sim 10^{10}\right)$.

For $T=10^{4} \mathrm{~K} \sim 1 \mathrm{eV}$ in the HII region [12] $\epsilon=10^{-2}[15-16]$ and $\lambda_{J}=3.09 \times 10^{8} \mathrm{~m}$ [17]. The physical strength of the lowest-order perturbed potential fluctuation (Figs. 1a-4a) for normalized potential fluctuation $\sim 10^{-6}$ is estmated as $\Phi_{\text {phys }} \sim \in\left(T_{p} \Phi_{1} / e\right) \sim 10^{-8} \mathrm{~V}$.

The profile of field fluctuations (Figs. 1b-4b) and potential curvatures (Fig. 1c-4c) show transition from damped periodic waves to a composite mixture of compressive and rarefactive 
soliton, and also, transition from non-periodic structure to a two-tail inverted bell-shaped soliton structure with increase in the grain-mass. The real strength of the electric field fluctuation is $E_{\text {phys }} \sim \in\left(T_{p} E_{e 1} / e \lambda_{J}\right) \sim 3.23 \times 10^{-18} \mathrm{Vm}^{-1}$. Again, the associated potential curvature fluctuation is $\left(\partial_{x x} \Phi\right)_{\text {phys }} \sim \in\left(T_{p} \partial_{\rho \rho} \Phi_{1} / e \lambda_{J}^{2}\right) \sim 1.04 \times 10^{-25} \mathrm{Vm}^{-2}$. This is very small quantity signifying that the global quasi-neutrality is not affected appreciably due to the weak perturbation treatment.

The phase portrait (Figs. 1d-4d) reveals that for the low grain-mass, the system is not conservative as the trajectories of the Columbic particles in the phase space is not closed curves. But, for high grain-mass (Fig. 3d), the phase portraits give information of the system to behave like a conservative one, as phase trajectories are closed curves. The phase portraits (Fig. 4d) show the transition of non-conservative system to conservative one with the increase in the grain-mass.

\section{V.2. Self-gravitational fluctuations}

The Jeans mode fluctuation dynamics of our idealized model is governed by the KdV equation (Eq. (75)) on the lowest-order perturbed self-gravitational potential $\left(\Psi_{1}\right)$. The dynamics is contributed jointly by all the species having inertial mass of non-zero value. To obtain the detailed characteristic features, the $\mathrm{KdV}$ equation without source is simulated numerically as an initial value problem as before. The numerical strength of the coefficients of convective and dispersive terms under some judicious plasma parameter values are $\left|A_{2}\right|=25.229$ and $\left|B_{2}\right|=0.288$, respectively. The various numerical profiles obtained are presented in Figs. 5-7.

Figure 5 shows the profile structures of the lowest-order perturbed self-gravitational (a) potential $\left(\Psi_{1}\right)$, (b) field $\left(-\Psi_{1 \rho}\right)$, (c) potential curvature $\left(\Psi_{1 \rho \rho}\right)$, and (d) phase portrait in the phase space defined by $\Psi_{1}$ and $\Psi_{1 \rho}$. Various lines correspond to Case (1): $m_{d}=1.67 \times 10^{-14} \mathrm{~kg}$ (blue line), Case (2): $m_{d}=2.17 \times 10^{-14} \mathrm{~kg}$ (red line), Case (3): $m_{d}=2.65 \times 10^{-14} \mathrm{~kg}$ (green line), and Case (4): $m_{d}=3.20 \times 10^{-14} \mathrm{~kg}$ (black line), respectively. Different input initial values used are $(\Psi)_{i}=0.00,\left(\Psi_{\rho}\right)_{i}=-1.00 \times 10^{-8}$, and $\left(\Psi_{\rho \rho}\right)_{i}=-2.90 \times 10^{-5}$. The other parameters kept fixed are $m_{e}=9.109 \times 10^{-31} \mathrm{~kg}, m_{i}=1.673 \times 10^{-27} \mathrm{~kg}, n_{e o}=$ $2.00 \times 10^{12} \mathrm{~m}^{-3}, n_{i o}=4.15 \times 10^{12} \mathrm{~m}^{-3}, n_{\text {dno }}=4.00 \times 10^{3} \mathrm{~m}^{-3}, n_{d c o}=1.00 \times 10^{10} \mathrm{~m}^{-3}$, $\mu=4.00 \times 10^{-2}, F_{e d}=10.00$, and $F_{i d}=1.00$. It is observed that the self-gravitational fluctuations evolve as inverted bell-shaped (rarefactive) soliton-like eigenmodes (Fig. 5a). The amplitude of the solitary patterns decreases with increase in grain-mass, and the negative peak of the soliton shifts outwards relative to the entre. When the mass increases, the self-gravitational temptation of the DMC also increases, which assists the decrease of the fluctuation amplitude. The corresponding eigenmode fields exhibit hybrid structures composed of compressive and rarefative soliton pairs (Fig. 5b). The associated curvature shows peakon-like structures, which depict that the deviation from gravitational mass-neutrality is maximum at the radial point $\rho \sim 5.50$ on the Jeans length (Fig. 5c).The entire dynamics, however, is a conservative one as shown by the closed-form structures in the phase trajectories with the most stable fixed point at $(0,0)$ in the defined phase space (Fig. 5d).

Figs. 6 and 7 sketch same as Fig. 5, but under different conditions. Profiles in Fig. 6 show that the amplitude of inverted bell-shaped soliton-like (rarefactive) eginenmodes decreases with increase in equilibrium value of neutral dust-grain population. The underlying physics is that the self-gravitational attractive force between the neutral particles increases with the increase in 


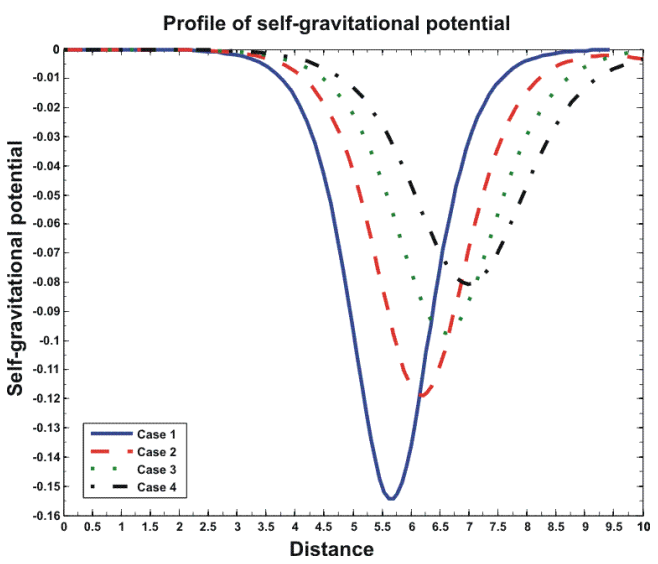

(a)

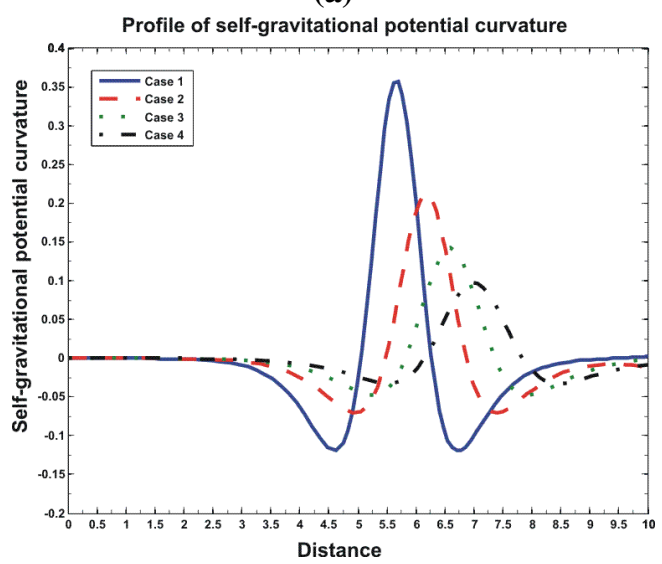

(c)

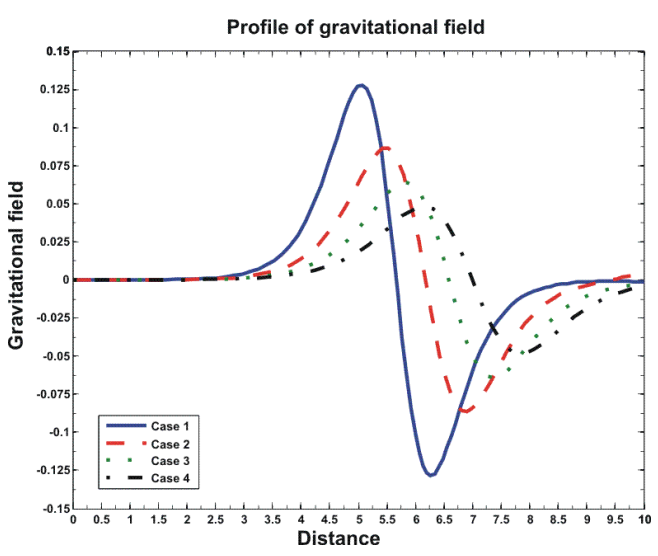

(b)

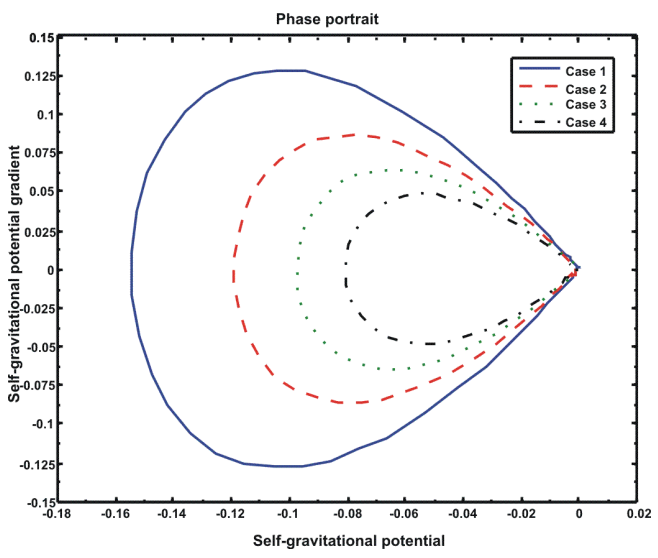

(d)

Fig. 5. Profile of the lowest-order perturbed self-gravitational (a) potential, (b) field, (c) potential curvature, and (d) phase portrait. Various lines correspond to Case (1): $m_{d}=$ $1.67 \times 10^{-14} \mathrm{~kg}$ (blue line), Case (2): $m_{d}=2.17 \times 10^{-14} \mathrm{~kg}$ (red line), Case (3): $m_{d}=2.65 \times 10^{-14} \mathrm{~kg}$ (green line), and Case (4): $m_{d}=3.20 \times 10^{-14} \mathrm{~kg}$ (black line), respectively. Different fixed and input initial values used are defined in the text.

the neutral grain density, which tries to gravitationally bind the particles more tightly. Thus, selfgravitationally bounded compactness lowers the fluctuation amplitude. Similarly, profiles in Fig. 7 reveal that the magnitude of the wave amplitude increases with increase in equilibrium charged dust density. With increase in the equilibrium value of the charged dust population, the repulsive force increases, this counteracts the self-gravitational attractive forces. As a result, it makes small increase in the amplitude of the self-gravitational fluctuations, although more negative in sense. Rest of the dynamical features of the eigenmode spectrum evolves accordingly as highlighted before.

The following are some other main points of astrophysical attention, which may be drawn from the analyzed self-gravitational fluctuation dynamics. 


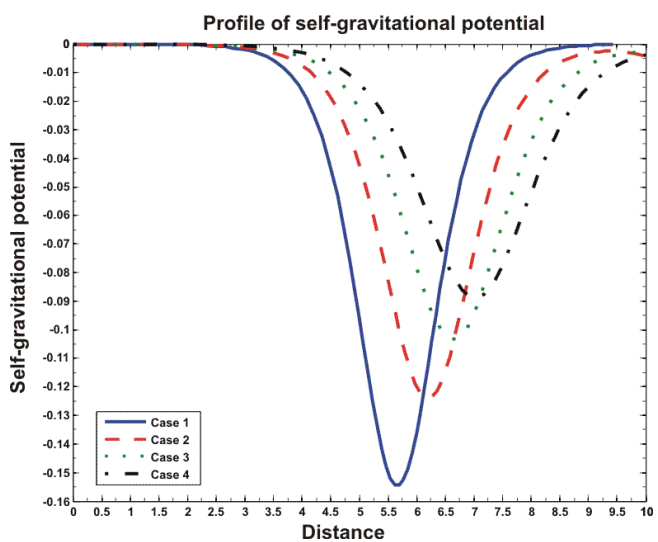

(a)

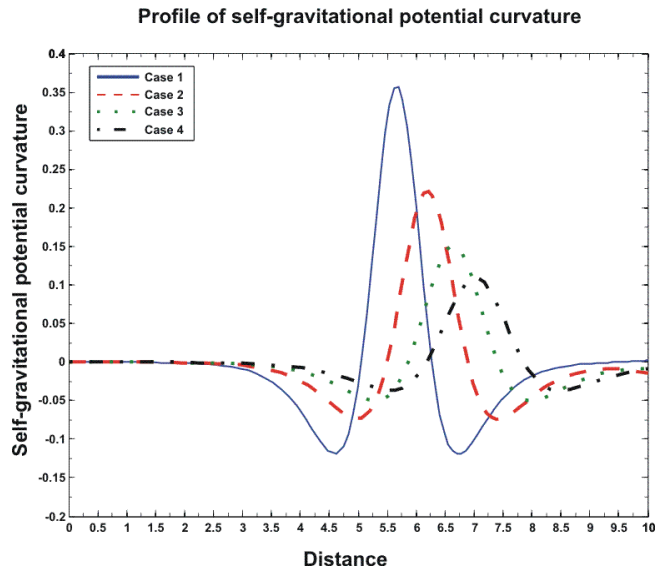

(c)

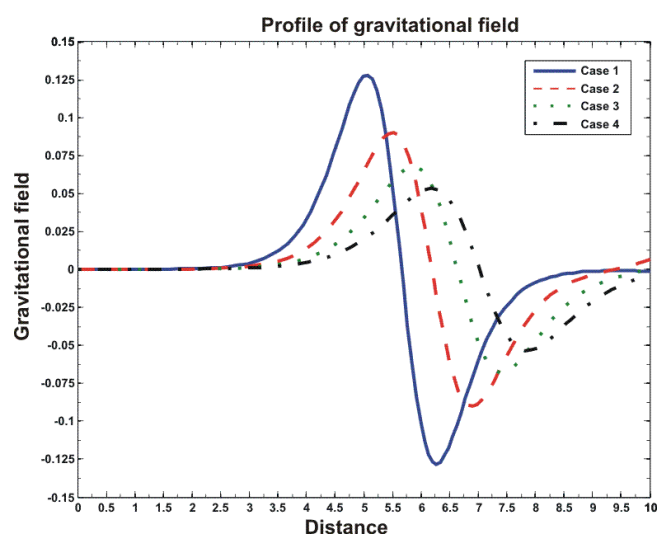

(b)

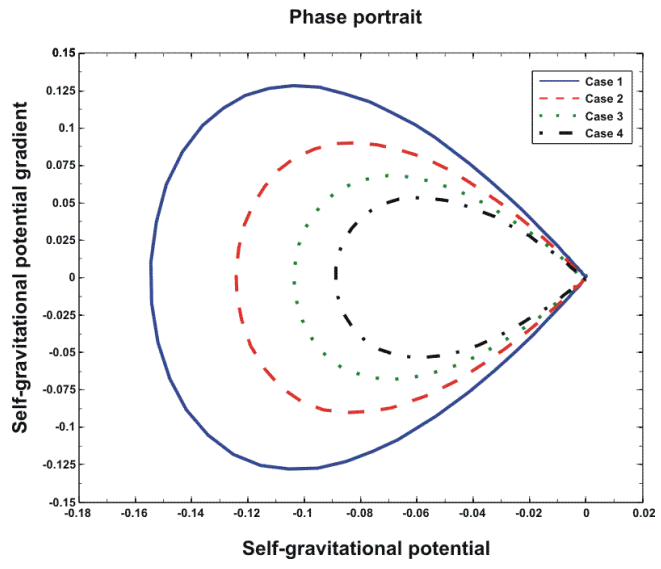

(d)

Fig. 6. Same as Fig. 5, but with $m_{d}=1.67 \times 10^{-14} \mathrm{~kg}$. Various lines correspond to Case (1): $n_{d n o}=4.00 \times 10^{3} \mathrm{~m}^{-3}$ (blue line), Case (2): $n_{\text {dno }}=5.00 \times 10^{3} \mathrm{~m}^{-3}$ (red line), Case (3): $n_{d n o}=6.00 \times 10^{3} \mathrm{~m}^{-3}$ (green line), and Case (4): $n_{d n o}=7.00 \times 10^{3} \mathrm{~m}^{-3}$ (black line), respectively.

1) The lowest-order self-gravitational potential fluctuations appear as inverted bell-shaped soliton-like (rarefactive) eigenmodes (Figs. 5a-7a). Their signatures have also been reported in the past as observed by various multispace satellites like Freja, Viking, etc [13,18].

2) For $T=10^{4} \mathrm{~K} \sim 1 \mathrm{eV}$ in the HII region [12] and $\in=10^{-2}$ [15-17] the physical strength of the self-gravitational fluctuations for $\Psi_{1} \sim 10^{-2}$ is equivalent to $\left|\Psi_{\text {phys }}\right| \sim \in\left(T_{p} \Psi_{1} / e\right) \sim 10^{-4}$ $\mathrm{V}$.

3) The self-gravitational field profiles (Figs. 5b-7b) show the eigenmodes, which are composite mixtures of compressive and rarefactive solitons, i. e., admixtures of solitons and antisolitons. The real strength of the field fluctuations is equivalent to $\left|E_{\text {gphys }}\right| \sim \in\left(T_{p} E_{G 1} / e \lambda_{J}\right) \sim$ $3.23 \times 10^{-13} \mathrm{Vm}^{-1}$ for $\lambda_{J}=3.09 \times 10^{8} \mathrm{~m}[21,22]$. 


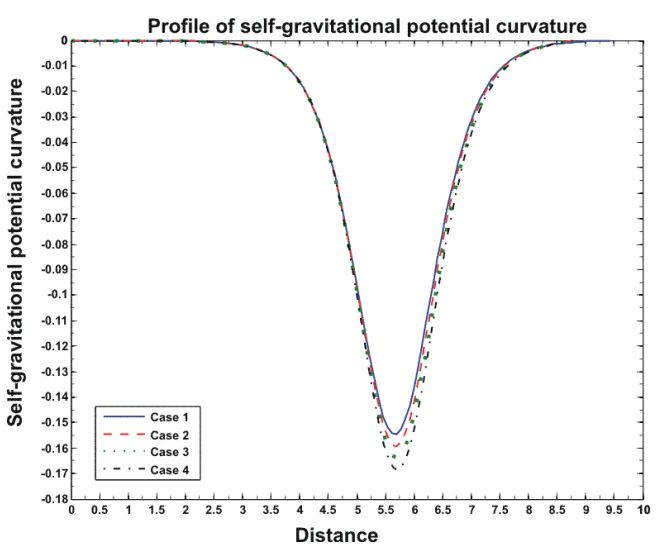

(a)

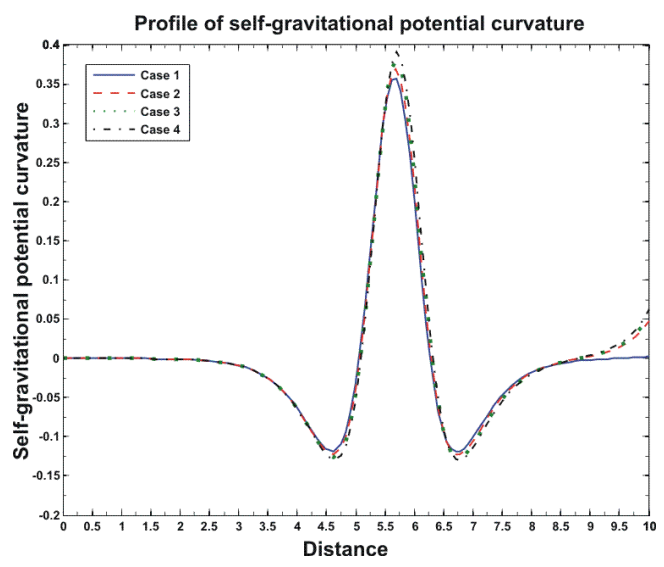

(c)

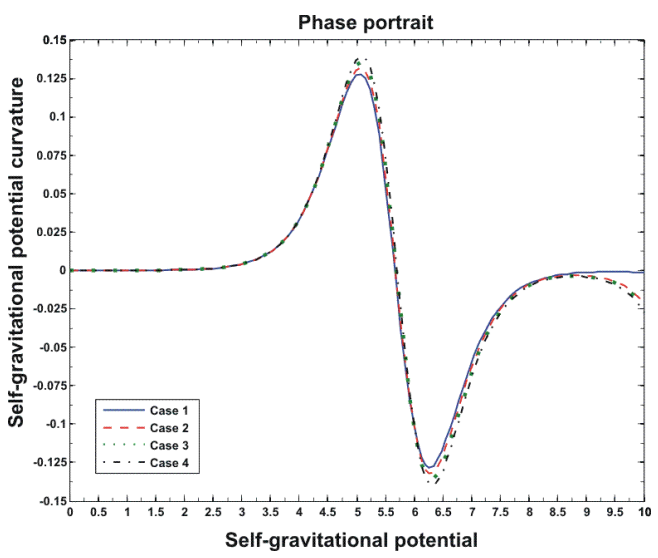

(b)

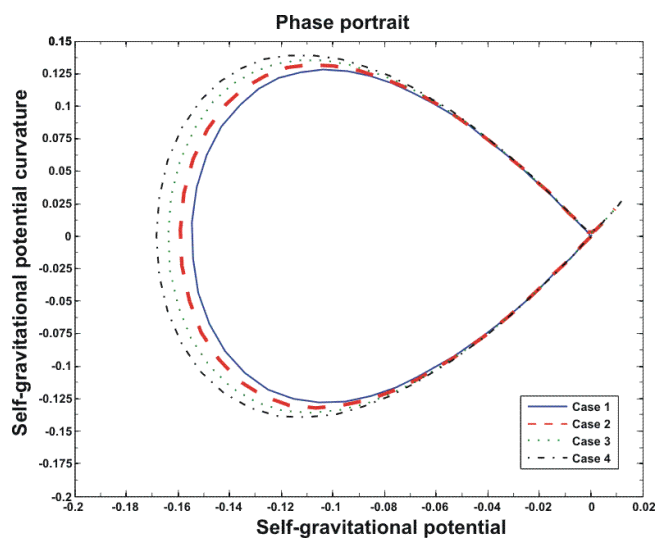

(d)

Fig. 7. Same as Fig. 5, but with $m_{d}=1.67 \times 10^{-14} \mathrm{~kg}$. Various lines correspond to Case (1): $n_{d c o}=1.00 \times 10^{10} \mathrm{~m}^{-3}$ (blue line), Case (2): $n_{d c o}=1.03 \times 10^{10} \mathrm{~m}^{-3}$ (red line), Case (3): $n_{d c o}=1.06 \times 10^{10} \mathrm{~m}^{-3}$ (green line), and Case (4): $n_{d c o}=1.09 \times 10^{10} \mathrm{~m}^{-3}$ (black line), respectively.

4) The potential curvature profiles (Figs. 5c-7c) appear like solitary patterns. Its real magnitude is equivalent to $\left|\left(\partial_{x x} \Psi\right)_{\text {phys }}\right| \sim \in\left(T_{p} \partial_{\rho \rho} \Psi_{1} / e \lambda_{J}^{2}\right) \sim 1.04 \times 10^{-20} \mathrm{Vm}^{-2}$. This is very small quantity signifying that the global mass-neutrality is not affected appreciably due to the lowest-order nonlinearity in the fluctuations.

The phase portraits (Figs. 5d-7d), which give the geometrical representation of the trajectories of the Newtonian particles as closed curves; show that the self-gravitating dynamics under the considered judicious situation is a conservative one.

\section{CONCLUSIONS}

We try to study the properties of the nonlinear excitation and propagation of gravitoelectrostatic fluctuations (both electrostatic and Jeans modes) in a field-free self-gravitating DMC 
with diverse plasma inhomogeneities within a planar geometry. It is based on the Jeans assumption of homogenizing self-gravitating plasma. The unique originality of this articulation is the inclusion of all the realistic possible factors encountered by the different constituent species in hydrodynamic configuration simultaneously. Weak but finite thermal inertia, nonlinear flowconvection, grain-charge fluctuation and all the realistic collisions are included in the model amid non-equilibrium plasma inhomogeneities simultaneously. It is seen that the gravito-electrostatic fluctuations are collectively governed by a unique pair of $\mathrm{KdV}$ equations obtained by multiple scaling techniques. A wide spectrum of shock-soliton patterns is found to exist in the cloud numerically. The effect of diverse plasma parameters on the amplitude and structures of the eigenmodes is investigated. We explicitly find the windows in plasma parameter space, where a unique interesting transition from oscillatory shock (with smaller average amplitude) to soliton (with larger average amplitude) is specifically supported. Based on our stability analysis, the following main conclusive remarks of astrophysical significance, importance and implication may be summarized.

1) The nonlinear gravito-electrostatic fluctuations of a planar DMC are governed by a unique pair of $\mathrm{KdV}$ equations obtained by multicale analysis under the framework of the point charge (Coulombic) and point mass (Newtonian) approximations within the non-relativistic limit.

2) The lowest-order electrostatic potential fluctuations co-evolve as driven damped oscillatory shock (for low dust mass) and two tail compressive soliton-like eigenmodes (for high dust mass). The driving nature is attributed due to the presence of the self-consistent nonlinear source in the governing electrostatic $d-\mathrm{KdV}$ equation. The eigenmode structures are in good correspondence with the experimentally detected shocks in collision-dominated plasma [10-11]; multispace satellite observations like Freja, Viking, etc [5,7,13] and analytical findings by others [27-33]. On the other hand, the self-gravitational fluctuation counterparts governed by the KdV equation appear as inverted bell-shaped (rarefactive) solitary spectral patterns.

3) The variation of dust charge density resulting from propagation of the gravito-electrostatic waves is significant for astrophysical context. The condensation of the grains due to the propagation of the gravito-electrostatic shocks and solitons through interstellar media enhances the self-gravitational interaction, which is a feasible process for star and planet formation [6-18].

4) An increase in the grain-mass makes the self-gravitational effects more effective. So, the self-gravitational dispersive process dominates internal dissipative process (dust-charge variation and collision) amid pulsating gravito-electrodynamic coupling, which generates the unique transition of oscillatory shock-to-soliton.

5) The geometrical trajectories of the Columbic particles in the phase space show that the system is non-conservative for low dust-mass, and conservative for high grain-mass. Again, the trajectories of the Newtonian particles in the phase space depict that the self-gravitational dynamics, in isolation, with specific judicious initial input values, is of conservative nature.

6) It is found by comparing the electrostatic and self-gravitational fluctuations $\left(\Phi_{\text {phy }} /\left|\Psi_{\text {phy }}\right| \sim 10^{-4}\right)$ that the self-gravitational fluctuations are more dominant than the electrostatic counterparts due to the presence of the heavier grains in the cloud.

7) Finally, the adopted methodologies, strategic techniques and analyses may extensively be useful as the input elements in investigating the basic features of the self-gravitational collapse, formation and evolution of stars, galactic structures and other cluster-like astrophysical objects in different practical regimes of space and plasma environments. Besides, the associated wave 
dynamics in presence of realistic fields with spatiotemporal variation of the equilibrium in presence of full nonlinearities in higher dimensions [33] is an interesting investigation of astrophysical importance, which is in progress for future communication elsewhere.

\section{ACKNOWLEDGEMENTS}

The financial support from the Department of Science and Technology (DST) of New Delhi, Government of India, extended to the authors through the SERB Fast Track Project (Grant No. SR/FTP/PS-021/2011) is thankfully recognized.

\section{REFERENCES}

[1] M. Khan, S. Ghosh, S. Sarkar and M. R. Gupta, Physica Scripta T116 (2005) 53.

[2] A. A. Mamun and P. K. Shukla, IEEE Transactions on Plasma Science 30 (2002) 720.

[3] P. K. Shukla, V. P. Silin, Physica Scripta 45 (1992) 508.

[4] J. Vranjes, B. P. Pandey and S. Poedts, Physical Review E 64 (2001) 066404.

[5] S. Burman and A. R. Chowdhury, Chaos, Solitons \& Fractals 13 (2002) 973.

[6] M. R. Gupta, S. Sarkar, S. Ghosh, M. Debnath and M. Khan, Physical Review E 63 (2001) 046406.

[7] G. Zhi-Rong, Y. Zeng-Quiang, Y. Bao-Xiang and S. Mao-Zhu, Chinese Physics B 19 (2010) 115203.

[8] N. N. Rao and P. K. Shukla, Planetary \& Space Science 42 (1994) 221.

[9] A. Barkan, R. L. Marlino and N. D. Angelo, Physics of Plasmas 2 (1995) 3563.

[10] R. L. Marlino, J. R. Heinrich, S-H Hyun and J. K. Meyer, Physics of Plasmas 19 (2012) 057301.

[11] Y. Nakamura, H. Bailung and P. K. Shukla, Physical Review Letters 83 (1999) 1602.

[12] B. P. Pandey, J. Vranjes, S. Poedts and P. K. Shukla, Physica Scripta 65 (2002) 513.

[13] F. Verheest, Space Science Reviews 77 (1996) 267.

[14] F. Verheest and V. M. Cadez, Physical Review E 66 (2002) 056404.

[15] C. B. Dwivedi, A. K. Sen and S. Bujarbarua, Astronomy \& Astrophysics 345 (1999) 1049.

[16] P. K. Karmakar, Pramana- Journal of Physics 76 (2011) 945.

[17] P. K. Karmakar and B. Borah, Physica Scripta 86 (2012) 025503.

[18] T. Cattaert and F. Verheest, Astronomy \& Astrophysics 438 (2005) 23.

[19] R. S. Klessen, M. R. Krumholz, and F. Heitsch, Advance Science Letter 4 (2011) 258.

[20] K. Avinash and P. K. Shukla, Physics Letters A 189 (1994) 470.

[21] P. K. Karmakar and B. Bora, European Physics Journal D 67 (2013) 187.

[22] P. K. Karmakar and B. Bora, Plasma Science \& Technology 2013 (accepted).

[23] C. B, Dwivedi and R. Prakash, Journal of Applied Physics 90 (2001) 3200.

[24] P. K. Karmakar, U. Deka and C. B. Dwivedi, Physics of Plasmas 12 (2005) 032105.

[25] P. K. Karmakar, U. Deka and C. B. Dwivedi, Physics of Plasmas 13 (2006) 104702.

[26] U. Deka and C. B. Dwivedi, Brazilian Journal of Physics 40 (2010) 333.

[27] U. Deka, A. Sarma, R. Prakash, P. K. Karmakar and C. B. Dwivedi, Physica Scripta 69 (2004) 303.

[28] S. Mahmood and H. Ur-Rehman, Physics of Plasmas 17 (2010) 072305.

[29] A. V. Volosevich and C-V. Meister, Contribution to Plasma Physics 42 (2012) 61.

[30] S. I. Popel, T. V. Losseva, A. P. Golub, R. L. Merlino and S. N. Andreev, Contribution to Plasma Physics 45 (2005) 461.

[31] J. Vranjes, Astrophysics \& Space Science 213 (1994) 139.

[32] F. Verheest and P. K. Shukla, Physica Scripta 55 (1997) 83.

[33] H. R. Pakzad and K. Javidan Chaos, Solitons \& Fractals 42 (2009) 2904 